Draft Version July 2, 2019

Preprint typeset using $\mathrm{LAT}_{\mathrm{E}} \mathrm{X}$ style emulateapj v. 12/16/11

\title{
INFRARED CONTRIBUTIONS OF X-RAY SELECTED ACTIVE GALACTIC NUCLEI IN DUSTY STAR-FORMING GALAXIES
}

\author{
Arianna Brown ${ }^{1,2}$, Hooshang Nayyeri ${ }^{1}$, Asantha Cooray ${ }^{1}$, Jingzhe MA ${ }^{1}$, Ryan C. Hickox ${ }^{3}$, And Mojegan Azadi ${ }^{4}$ \\ ${ }^{1}$ Department of Physics and Astronomy, University of California, Irvine, CA 92697 \\ ${ }^{2}$ Department of Physics and Astronomy, California State University, Los Angeles, CA 90032, USA \\ ${ }^{3}$ Department of Physics and Astronomy, Dartmouth College, Hanover, NH 03755, USA and \\ ${ }^{4}$ Harvard-Smithsonian Center for Astrophysics, 60 Garden Street, Cambridge, MA, 02138, USA \\ Draft version July 2, 2019
}

\begin{abstract}
We investigate the infrared contribution from supermassive black hole activity versus host galaxy
\end{abstract} emission in the mid to far-infrared (IR) spectrum for a large sample of X-ray bright active galactic nuclei (AGN) residing in dusty, star-forming host galaxies. We select 703 AGN with $L_{\mathrm{X}}=10^{42}-$ $10^{46}$ ergs s $^{-1}$ at $0.1<z<5$ from the Chandra XBoötes X-ray Survey with rich multi-band observations in the optical to far-IR. This is the largest sample to date of X-ray AGN with mid and far-IR detections that uses spectral energy distribution (SED) decomposition to determine intrinsic AGN and host galaxy infrared luminosities. We determine weak or nonexistent relationships when averaging starformation activity as a function of AGN activity, but see stronger positive trends when averaging $L_{\mathrm{X}}$ in bins of star-forming activity for AGN at low redshifts. We estimate an average dust covering factor of $33 \%$ based on infrared SEDs and bolometric AGN luminosity, corresponding to a Type 2 AGN population of roughly a third. We also see a population of AGN that challenge the inclination based unification model with individual dust covering factors that contradict the nuclear obscuration expected from observed X-ray hardness ratios. We see no strong connection between AGN fractions in the IR and corresponding total infrared, $24 \mu \mathrm{m}$, or X-ray luminosities. The average rest-frame AGN contribution as a function of IR wavelength shows significant $(\sim 80 \%)$ contributions in the mid-IR that trail off at $\lambda>30 \mu \mathrm{m}$. Additionally, we provide a relation between observed $L_{\mathrm{X}}$ and pure AGN IR output for high-z AGN allowing future studies to estimate AGN infrared contribution using only observed X-ray flux density estimates.

Keywords: Galaxies: active — Galaxies: nuclei — Galaxies: evolution — Infrared: galaxies — X-rays: galaxies

\section{INTRODUCTION}

Nearly all massive galaxies are believed to host a supermassive black hole (SMBH) at their center (Kormendy \& Richstone 1995; Magorrian et al. 1998; Ho 2008). Current research suggests that central black holes gain mass through a combination of both coalescence and bursts of mass accretion from the environment as the host galaxy evolves (Kauffmann \& Haehnelt 2000; Volonteri et al. 2003; Somerville et al. 2008; Shankar 2009; Volonteri 2010, and references therein). The peak epoch of central black hole accretion, as the main source of active galactic nuclei (AGN), coincides with the peak epoch of star-formation in the universe at $\mathrm{z} \approx 1-2$ (Di Matteo et al. 2005; Lutz et al. 2008; Aird et al. 2010; Stevens et al. 2010; Bonfield et al. 2011; Alexander \& Hickox 2012), and also major galaxy merger events (Di Matteo et al. 2005; Hopkins \& Hernquist 2009; Treister et al. 2012; Ellison et al. 2013; Rosario et al. 2015). Furthermore, in our local universe there exists a tight correlation between SMBH mass and host galaxy bulge mass and stellar velocity dispersions (Ferrarese \& Merritt 2000; Marconi \& Hunt 2003; Gültekin et al. 2009; Kormendy \& Ho 2013, and references therein), whereas higher redshift SMBHs have been found in smaller host galaxies than expected (e.g. Shields \& Salviander 2009, and references therein). These results signify that SMBH growth and galaxy growth are co-evolutionary processes and that these processes may regulate each other over time to pro- duce the galaxy and SMBH sizes we observe today.

Both central black hole growth and star formation rely on the abundance of cold molecular gas (Croton et al. 2006; Smith et al. 2008; Dekel et al. 2009; Dijkstra \& Loeb 2009; Bonfield et al. 2011). While cold dust and gas collapse to trigger star formation, the $\mathrm{SMBH}$ at the galaxy core gravitationally attracts cold gas and dust into a clumpy obscuring reservoir a few parsecs out from the SMBH, which fuels a thin, hot SMBH accretion disk with a radius typically $\lesssim 1$ parsec (Antonucci 1993; Tristram et al. 2007, 2009; Hopkins et al. 2012; Davies et al. 2015). The AGN feeds off the reservoir (hereby referred to as a torus; although it is now accepted that the dust is distributed in a more clumpy manner as opposed to a smooth donut structure (Nenkova et al. 2008a,b; Siebenmorgen et al. 2015)) with a mass accretion process that emits X-ray, UV, and optical light (e.g. see Haardt \& Maraschi 1991). The X-ray, UV and optical light is partially absorbed by the surrounding dusty toroidal structure, then re-emitted in the infrared, making most AGN bright in the mid-IR, but not all AGN are X-ray bright (e.g. Treister et al. 2004; Stern et al. 2005; Daddi et al. 2007; Donley et al. 2012). The current AGN unified model posits that AGN can be classified by the orientation of the dusty torus to the observer's line of sight (Antonucci 1993; Urry \& Padovani 1995): Type 1 AGN are usually observed face-on through a cavity in the torus and are typically bright in the X-ray, UV and optical 
spectrum; Type 2 AGN may be intrinsically less luminous or are observed at an angle through the torus, and are thereby obscured by high column densities of dust and gas $\left(N_{H}>1.5 \times 10^{24} \mathrm{~cm}^{-2}\right)$ from the observer's lineof-sight, enough so that most or all of the X-ray emission is absorbed and undetected (e.g. Aird et al. 2012; Lanzuisi et al. 2015). However, recent observations are challenging this scheme (e.g. see section 3.1 of Bianchi et al. 2012) and suggesting that observational differences in obscuration between AGN are mostly driven by individual SMBH accretion rates (e.g. Lusso et al. 2012; Ricci et al. 2017) or host galaxy obscuration (e.g. Goulding et al. 2012; Netzer 2015; Chen et al. 2015; Hickox \& Alexander 2018).

AGN accretion and outflow mechanisms are theorized to play a major role in galaxy evolution, via heating up, consuming and/or blasting away the host galaxy's remaining cold gas and dust necessary to create new stars, thereby triggering a star-formation quenching phase (Di Matteo et al. 2005; Harrison et al. 2014; Hopkins et al. 2006; Fabian 2012, and references therein). In observations, some AGN feedback processes are instantaneously strong enough to affect star formation in the host galaxy (e.g. Sturm et al. 2011; Reeves et al. 2009; Rupke \& Veilleux 2011, but also see Leung et al. 2017); however, the exact contribution of the AGN phase to the physical properties of galaxies, compared to other mechanisms from stellar processes, is still not well understood (e.g. Silk \& Nusser 2010; Geach et al. 2014; Diamond-Stanic et al. 2012; Gabor \& Bournaud 2014), particularly for the most powerful AGN (e.g. Stanley et al. 2015; Rosario et al. 2012). To study the effect of powerful AGN on their host galaxies, it is necessary to have a large statistical sample of AGN with multi-band observations to individually derive and constrain their physical properties.

One of the main degeneracies in determining the evolutionary relationship between AGN and host galaxy starformation lies in their mutual obscuration by warm dust (Delvecchio et al. 2015; Symeonidis et al. 2016; Lutz et al. 2008). The radiation originating from warm dust in stellar nebulae and from the obscuring torus around AGN are both bright in the mid to far-IR spectrum and thus necessary to disentangle prior to using IR radiation as an indicator for any host galaxy dust properties, including measurements of dust temperatures, host galaxy stellar mass, and star-formation rates; without this decomposition, there is a risk of measurement overestimation and, therefore, an increase in uncertainties. AGN accretion and outflow mechanisms release a large amount of energy detectable at nearly all wavelengths, in particular $\mathrm{X}$-rays from the accretion disk (see Brandt \& Alexander 2015, for a review of AGN viewed in the X-ray spectrum) and radio signatures from synchroton radiation (e.g. Miley 1980; Blandford \& Payne 1982; Jorstad et al. 2005; Condon et al. 1995). These features are the most commonly utilized as identifiable signatures that could be used to distinguish AGN from their host galaxies (Donley et al. 2005; Del Moro et al. 2013; Mushotzky 2004; Brandt \& Alexander 2015).

Observational studies and models of IR SEDs for local AGN reveal radiative flux densities that generally increase through the mid-IR then rapidly decline starting somewhere between $40 \mu m<\lambda<100 \mu \mathrm{m}$ out to sub-

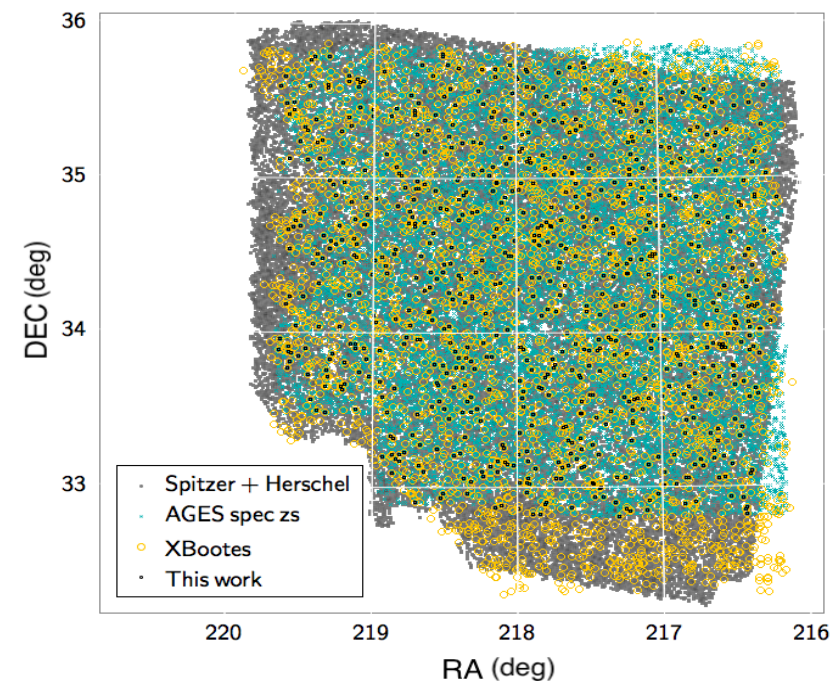

Figure 1. Survey map for the parent surveys from which our main sample is derived. Grey points mark all galaxies with a $\mathrm{S} / \mathrm{N}$ $>3$ in the MIPS $24 \mu m$ band and at least one SPIRE band; blue points denote all galaxies in the AGES survey with spectroscopic redshifts; gold circles outline all of the X-ray sources in the XBoötes survey; black points mark our final sample of 703 AGN and host galaxies which spans $\sim 7 \mathrm{deg}^{2}$. Respective survey coverage and depths are discussion in Section 2.

millimeter wavelengths (Mullaney et al. 2011). Prior to the Herschel Space Observatory (Pilbratt et al. 2010), observations were limited out to $\lambda<200 \mu \mathrm{m}$ only for a small sample of very far-IR bright, mostly local objects (e.g. Omont et al. 2001; Haas et al. 2003). Herschel has been instrumental in constraining the dust SEDs for large samples of local and high redshift AGN and star forming galaxies, revealing a universe that is optically obscured by dust and therefore undetected at shorter wavelengths (e.g. Mullaney et al. 2015; Symeonidis et al. 2016; Casey et al. 2014, and references therein).

In this paper, we use multi-wavelength infrared observations from the Herschel Space Observatory (Griffin et al. 2010; Poglitsch et al. 2010) combined with the Spitzer Space Telescope (Werner et al. 2004), along with optical wide-area observations, and X-ray data from the Chandra X-ray Observatory (Weisskopf et al. 2002) to construct the AGN and host galaxy SEDs and explore the warm dust properties in the context of AGN accretion activity. We focus on X-ray selected AGN in the wide $9.3 \mathrm{deg}^{2}$ Boötes legacy field (Jannuzi \& Dey 1999) with mid and far-IR counterparts detected by Herschel and Spitzer (Oliver et al. 2012; Ashby et al. 2009). The rich amount of data in the IR allows us to avoid the uncertainties that arise from single-band SED fitting. Furthermore, the multi-wavelength detections allow us to reliably use SED decomposition models to isolate AGN contribution in the infrared, reducing the likelihood of AGN contamination when estimating host galaxy properties.

This paper is organized as follows. Section 2 describes the multi-wavelength survey data used in this analysis. Section 3 details the AGN sample selection procedure. In Section 4, we discuss the derivation of AGN and host galaxy properties and the results in the context of other published studies; section 5 provides a summary of this work. Throughout this study, we assume a cosmology 


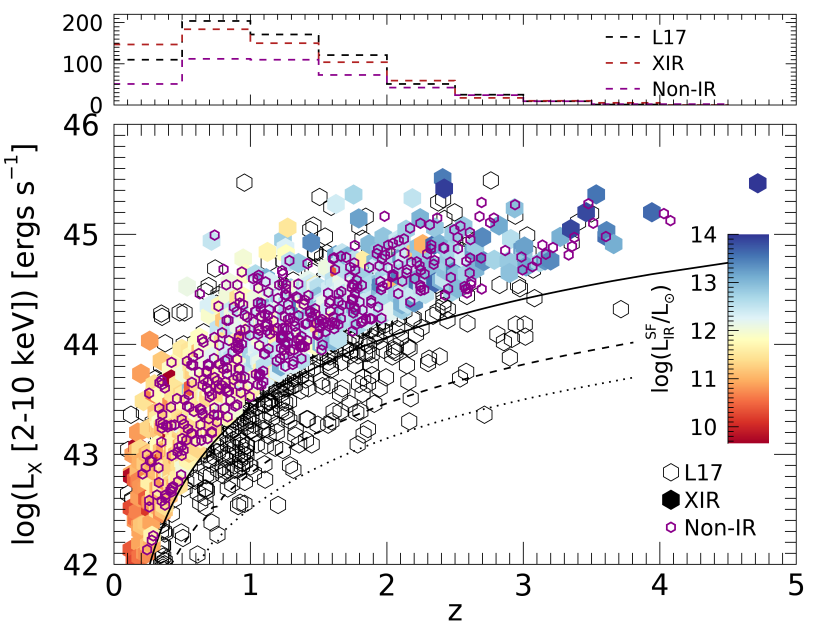

Figure 2. Distribution of rest-frame X-ray luminosities and spectroscopic redshifts for our AGN sample. The solid circles are the 703 X-ray AGN with Spitzer $24 \mu \mathrm{m}$ and far-infrared Herschel detections. Colors represent rest-frame, infrared luminosities corrected for AGN contamination derived from individual respective SEDs (see Section 3). The purple circles are the 425 X-ray AGN without mid/far-IR detections. The black empty circles are the AGN used for analysis in L17. The black solid line represents the X-ray flux limit of the Chandra XBoötes survey (Murray et al. 2005 ); for comparison, the dashed and dotted lines mark the sensitivity limits of the XMM-Newton (Brusa et al. 2010) and Chandra (Civano et al. 2016) surveys in the COSMOS field, respectively. Shown in the top panel is the number of sources in our XIR sample (red), non-IR sample (purple), and in L17 (black) in redshift bins of size 0.5 .

with $H_{0}=70 \mathrm{~km} \mathrm{~s}^{-1}, \Omega_{m}=0.3$, and $\Omega_{\Lambda}=0.7$.

\section{MULTI-WAVELENGTH DATA}

The survey observations used in this study are centered in the Boötes field at $\alpha=14 \mathrm{~h} 30 \mathrm{~m} 05.71 \mathrm{~s}, \delta=+34^{\circ} 16^{\prime}$ $47^{\prime \prime} .5$ (Jannuzi \& Dey 1999). We use publicly available photometric catalogs ranging from optical to far-infrared wavelengths, complemented with X-ray data and spectroscopic redshifts, with known active galaxies (Ashby et al. 2009) and clusters of AGN (Brand et al. 2006). The multi-wavelength observations cover different areas across the Boötes field (see Figure 1). Table 1 summarizes the data used and respective approximate field coverage.

The wide-area XBoötes survey provides us with a unique opportunity to probe a large population of the most powerful AGN, half of which are also embedded in galaxy powerhouses with total infrared luminosities $\left(\mathrm{L}_{\mathrm{IR}}\right.$ ) greater than $10^{12} L_{\odot}$ (also known as ultra-luminous infrared galaxies or ULIRGs). Some weakly accreting AGN and AGN obscured by Compton thick hydrogen column densities $\left(N_{H}>1.5 \times 10^{24} \mathrm{~cm}^{-2}\right)$ may be missed by X-ray surveys (e.g. Aird et al. 2012; Lanzuisi et al. 2015). However, studies confirm no single waveband can be used to select a complete sample of AGN, and X-ray detections remain one of the most reliable identification methods (e.g. Barmby et al. 2006; Mendez et al. 2013; Ellison et al. 2016; Cowley et al. 2016; Azadi et al. 2017; Brandt \& Alexander 2015, and references therein).

\subsection{X-ray Data}

Our AGN sample is selected from the Chandra XBoötes Survey, a 5-ks X-ray survey of the $9.3 \mathrm{deg}^{2}$
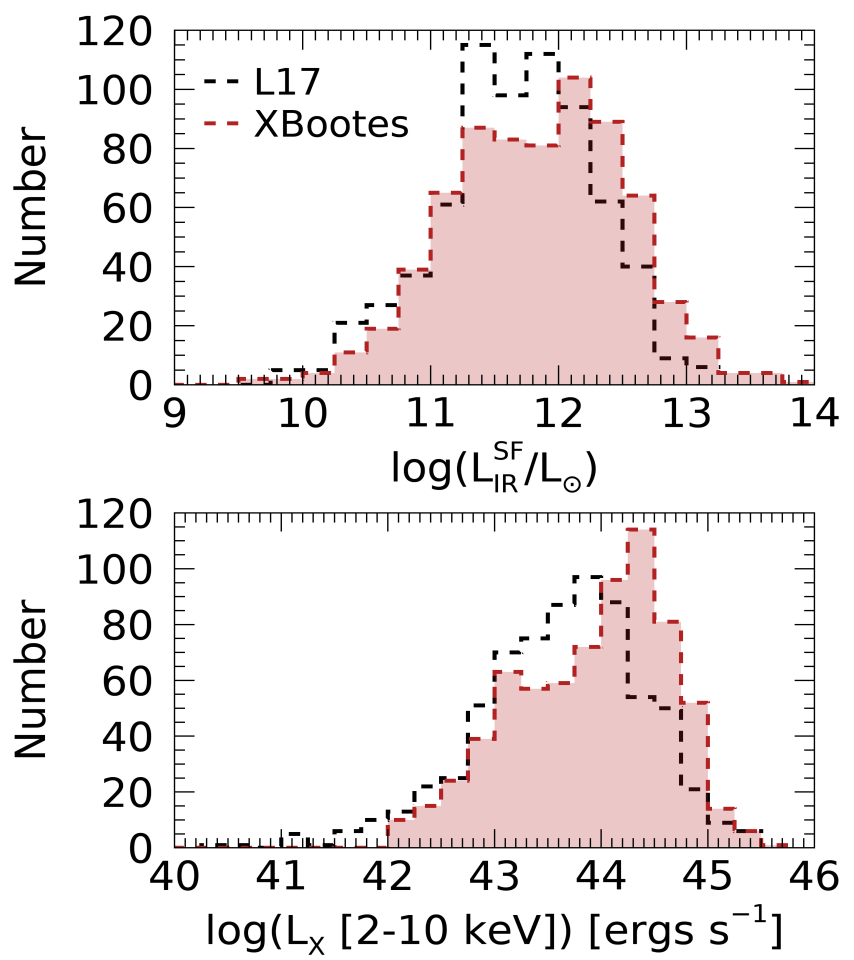

Figure 3. Distributions of AGN and host-galaxy properties comparing this sample (red) and Lanzuisi et al. (2017) (L17; black) samples. Top: Histogram of rest-frame, AGN-corrected infrared luminosities in bins of $1 \mathrm{dex}$, with median infrared luminosities of $1.95 \times 10^{45} \mathrm{ergs} \mathrm{s}^{-1}$ and $2.69 \times 10^{45} \mathrm{ergs} \mathrm{s}^{-1}$ for L17 and our sample, respectively. Bottom: Histogram of rest-frame X-ray luminosities in bins of 1 dex; our sample has a slightly higher median X-ray luminosity of $L_{\mathrm{X}}=1.07 \times 10^{44} \mathrm{erg} \mathrm{s}^{-1}$ compared to the L17 median X-ray luminosity of $L_{\mathrm{X}}=4.79 \times 10^{43} \mathrm{erg} \mathrm{s}^{-1}$.

Boötes Field as defined in the NOAO Deep Wide-Field Survey (NDWFS; Murray et al. 2005). This survey covers the full area defined by NDWFS with 126 individual $5 \mathrm{ks}$ contiguous pointings at uniform observational depths of $f_{0.5-7 \mathrm{keV}} \sim 8 \times 10^{-15} \mathrm{erg} \mathrm{s}^{-1} \mathrm{~cm}^{-2}$, yielding 3293 point sources with four or more counts. Rest-frame $\mathrm{X}$-ray luminosities are determined by the following equation (Alexander et al. 2003):

$$
\mathrm{L}_{\mathrm{X}}=4 \pi \times \mathrm{D}_{\mathrm{L}}^{2} \times \mathrm{F} \times(1+\mathrm{z})^{\Gamma-2}
$$

where $D_{L}$ is the luminosity distance, $F$ is the hard band $\mathrm{X}$-ray flux, $z$ is the redshift and a photon index of $\Gamma$ $=1.9$, which is typical for an unabsorbed X-ray luminous AGN (e.g. Vignali et al. 2005; Nandra \& Pounds 1994). To remain consistent in comparison to other studies, we translate our full band $0.5-7 \mathrm{keV}$ luminosities to $2-10 \mathrm{keV}$ hard band luminosities with a conversion factor of 0.78 , which is the ratio of respective intensities over each $\mathrm{keV}$ energy range for $\Gamma=1.9$. Due to the shallow nature of the XBoötes Survey, spectral fitting to correct for X-ray absorption is difficult or unachievable at an individual level for $\sim 90 \%$ of our sources (see Kenter et al. 2005; Murray et al. 2005, for a more detailed discussion), so we leave the observed fluxes to be interpreted at face value. We select sources with X-ray luminosities $\mathrm{L}_{\mathrm{X}}>10^{42} \mathrm{erg} \mathrm{s}^{-1}$ as lower luminosity sources may contain contamination from host galaxy processes (e.g. supernovae, X-ray binaries and massive stellar outflows; 
Table 1

Population counts and field coverage of the multi-wavelength flux catalogs used to generate 703 individual SEDs.

\begin{tabular}{llll}
\hline \hline Name & Bands & Survey Size & N Detected in Sample \\
\hline XBoötes Murray et al. (2005) & $0.5-7 \mathrm{keV}$ & $\sim 9.3 \mathrm{deg}^{2}$ & 703 \\
NDWFS Jannuzi \& Dey (1999) & $B_{w}, R, I$, and $K$ & $\sim 9.3 \mathrm{deg}^{2}$ & 652 \\
IR Boötes Imaging Survey Gonzalez & $H$ and $J$ & $\sim 9.3 \mathrm{deg}^{2}$ & $\sim 325$ \\
et al. (2010) & $3.6,4.5,5.8$ and $8.0 \mu \mathrm{m}$ & $\sim 10 \mathrm{deg}^{2}$ & $\sim 330$ \\
SDWFS Ashby et al. (2009) & $\sim 10 \mathrm{deg}^{2}$ & 703 \\
HerMES MIPS Oliver et al. (2012) & $24 \mu \mathrm{m}$ & $\sim 3 \mathrm{deg}^{2}$ & 138 and 181 \\
HerMES PACS Oliver et al. (2012) & 110 and $170 \mu \mathrm{m}$ & $\sim 8.5 \operatorname{deg}^{2}$ shallow, $\sim$ & 489,398, and 159 \\
HerMES SPIRE Oliver et al. (2012) & 250,300 and $500 \mu \mathrm{m}$ & $3 \mathrm{deg}^{2} \mathrm{deep}^{2}$ & \\
\hline
\end{tabular}

Ranalli et al. 2003; Mineo et al. 2012a,b; Lehmer et al. 2010). The X-ray survey depth of this study allows us to probe a larger population of the brighter end of the AGN luminosity function (see Figure 2). Figure 3 (bottom) displays the X-ray population distribution of this sample. The wider coverage of the XBoötes Survey allows us to study a large sample of powerful AGN with $50 \%$ of the 703 selected sources residing at or above $L_{X}$ $=1.07 \times 10^{44} \mathrm{ergs} \mathrm{s}^{-1}$; similar studies using surveys that may be deeper but cover smaller areas in the sky yield populations of weaker AGN; for example, Lanzuisi et al. (2017) (L17, hereafter) analyzed 692 X-ray selected AGN in the COSMOS field (Scoville et al. 2007) with a median $\mathrm{L}_{\mathrm{X}}=4.79 \times 10^{43} \mathrm{ergs} \mathrm{s}^{-1}$.

\subsection{Infrared Data}

Mid-IR and far-IR fluxes are collected from Data Release 4 of the Herschel Multi-tiered Extragalactic Survey $^{1}$ (HerMES; Oliver et al. 2012). Far-IR observations were taken by the Herschel Spectral and Photometric Imaging Receiver (SPIRE) at $250 \mu \mathrm{m}, 350 \mu \mathrm{m}$, and $500 \mu \mathrm{m}$ (Griffin et al. 2010), and the Herschel Photoconductor Array Camera and Spectrometer (PACS) $110 \mu \mathrm{m}$ and $170 \mu \mathrm{m}$ (Poglitsch et al. 2010) bands; mid-IR observations were completed by the Spitzer multi-band Imaging Photometer (MIPS) at $24 \mu \mathrm{m}$ (Rieke et al. 2004). Fluxes for all five Herschel bands used in the HerMES survey are recorded on positions defined by MIPS $24 \mu \mathrm{m}$ priors with a respective $5 \sigma$ detection limit at $\sim 0.3 \mathrm{mJy}$. The HerMES SPIRE campaign consisted of a combination of both deep and shallow observations: the center $\sim 3 \mathrm{deg}^{2}$ region is deeper and reaches $5 \sigma$ detection limits at $13.8,11.3$, and $16.4 \mathrm{mJy}$ at $250 \mu \mathrm{m}, 350 \mu \mathrm{m}$, and $500 \mu \mathrm{m}$, respectively; the outer $\sim 8.5 \mathrm{deg}^{2}$ region surrounding the center reaches $5 \sigma$ detection limits at 25.8 , 21.2 and $30.8 \mathrm{mJy}$ for the $250 \mu \mathrm{m}, 350 \mu \mathrm{m}$ and $500 \mu \mathrm{m}$ bands, respectively. The PACS observations occurred over the center $\sim 3 \mathrm{deg}^{2}$ of the Boötes region reaching $5 \sigma$ depths of 49.9 and $95.1 \mathrm{mJy}$ for the 110 and $170 \mu \mathrm{m}$ bands, respectively. Uncertainties in this analysis include both instrumental and confusion noise; we refer the reader to Roseboom et al. (2010) for a more detailed description of flux uncertainty determinations in the HerMES catalogs.

Near/Mid-IR catalogs were compiled from the Spitzer Deep, Wide-field Survey (SDWFS) (Ashby et al. 2009)

\footnotetext{
${ }^{1}$ http://hedam.oamp.fr/
}

which used all four channels of the Spitzer Infrared Array Camera (IRAC) (Fazio et al. 2004) to image the entire $\sim 10 \mathrm{deg}^{2}$ Boötes field. SDWFS is a combined four epoch survey that contains $\sim 10^{5}$ sources per band detected at $5 \sigma$ depths of $19.77,18.83,16.50$, and 15.82 Vega mag at $3.6 \mu \mathrm{m}, 4.5 \mu \mathrm{m}, 5.8 \mu \mathrm{m}$, and $8.0 \mu \mathrm{m}$, respectively. We also use $J$ and $H$-band data from the NEWFIRM Infrared Boötes Imaging Survey (Gonzalez et al. 2010) which reaches $5 \sigma$ limits of 22.05 and 21.30 Vega mag, respectively; and optical $B_{w}, R, I$ and $K$-band data from the NDWFS survey (Jannuzi \& Dey 1999) reaching $5 \sigma$ depths $^{2}$ at 26.6, 26.0, 26.0, and 21.4 AB mag, respectively. For all IR bands, we consider source detections at $>3 \sigma$.

\subsection{Redshifts}

Spectroscopic redshifts are extracted from the AGN and Galaxy Evolution Survey (AGES) (Kochanek et al. 2012), an optical spectroscopic and photometric redshift survey for optically selected sources in $7.7 \mathrm{deg}^{2}$ of the Boötes field. We limited our sample to spectroscopic redshifts in the range $z>0.1$ (Figure 2) to avoid the uncertainties associated with photometric redshifts and avoid contamination by local AGN and ULIRGs.

To investigate the evolution of AGN and galaxy properties with redshift, we complete our analysis over five redshift intervals and consider the X-ray - infrared relationship in each respective interval. The following redshift intervals are designed so that each interval has a sufficient number of sources $(\sim 90-200)$ to create several statistically significant bins within that range: $z=0.1-0.4,0.4-0.8,0.8-1.2,1.2-2$, and $2-5$. These redshift bins (z-bins) are consistent in comparison with several other similar studies, and contain 95, 178, 140, 195, and 95 sources, respectively.

\section{AGN SAMPLE SELECTION}

The final sample used in this study consists of powerful AGN with spectroscopically confirmed redshifts, and a detection in one Herschel SPIRE or PACS band. Since all objects in the HerMES campaign are based on Spitzer MIPS priors, it follows that every object in our sample has at least one $24 \mu \mathrm{m}$ detection as well as one Herschel detection. We achieve this sample, dubbed the XIR sample, through the following methods.

We matched X-ray AGN to infrared counterparts and spectroscopic redshifts using a nearest neighbor match-

2 https://www.noao.edu/noao/noaodeep/ 

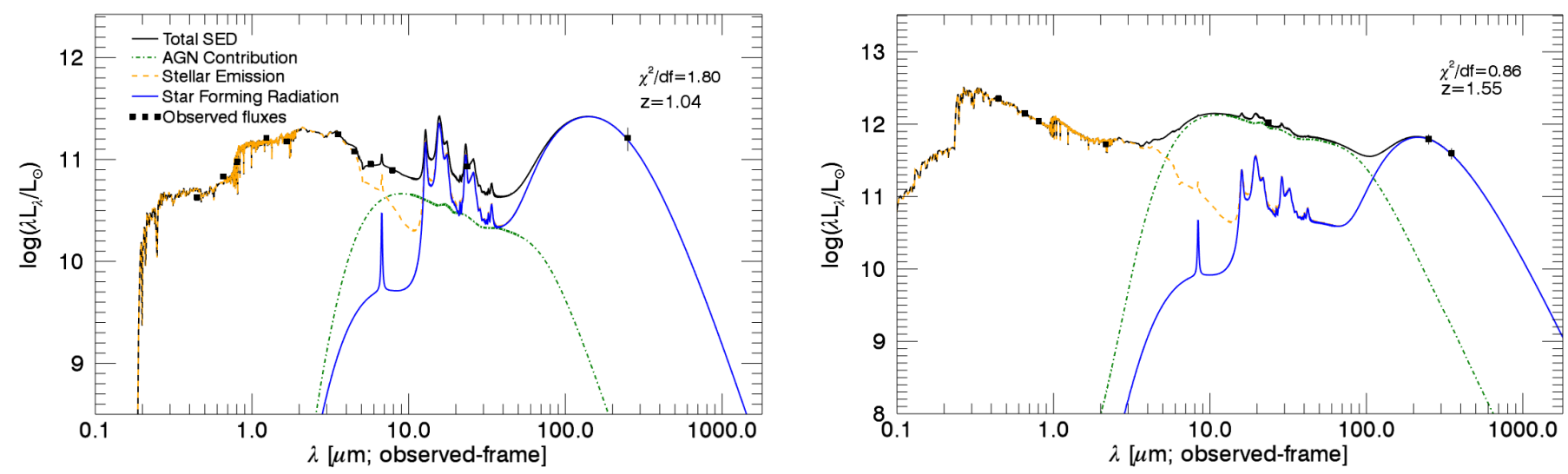

Figure 4. Example spectral energy distributions generated by SED3FIT (Berta et al. 2013). The dashed gold line is the stellar emission contribution, the blue line is the radiation contributed by star formation processes, the green dashed line is the contaminating radiation from the AGN including the heated dusty torus surrounding the black hole, and the black line is the total SED or the summation of the three components. Left: SED for a galaxy with star formation processes dominating the mid to far-infrared spectrum. Right: In this SED, the AGN component provides the most contribution in the mid-infrared (and some of the far-IR) spectrum that would typically be attributed to star formation processes.

ing technique. First, X-ray sources were matched to the AGES redshift catalog using a $1^{\prime \prime}$ search radius on their optical coordinates from Brand et al. (2006), with a spurious match rate estimated at $<1 \%$. We were able to use such a small search radius confidently due to prior work by Brand et al. (2006) who used a Bayesian matching scheme to determine optical counterparts for $98 \%$ of the X-ray sources in the XBoötes survey under a $1^{\prime \prime}$ search radius. We note that AGES redshifts were determined using optical spectroscopy, and as such this study explores the properties of brighter, less dust obscured active galaxies. We also note that the AGES survey misses $\sim 2 \mathrm{deg}^{2}$ of the XBoötes and HerMES survey (Figure 1), removing $10 \%$ of X-ray sources as possible candidates for this study. Near-IR and optical data were matched to the MIPS $24 \mu \mathrm{m}$ coordinates from the HerMES cata$\log$ (Oliver et al. 2012) using a $3^{\prime \prime}$ search radius, which corresponds to the Spitzer MIPS $24 \mu \mathrm{m}$ half width at half maximum. Finally, we matched the MIPS $24 \mu \mathrm{m}$ coordinates to the AGES coordinates. Again, we estimate a spurious match rate of $<1 \%$ when matching infrared data together, and once more when matching infrared data to X-ray sources with spectroscopic redshifts.

Prior to fitting a spectral energy distribution, we require an object to have a $24 \mu \mathrm{m}$ detection and a detection in one of the Herschel bands. The far-IR survey was defined on the coordinates for sources detected at $24 \mu \mathrm{m}$, thus any Boötes source detected by Herschel will also have a measurement at $24 \mu \mathrm{m}$. Even though Herschel observational depths varied across the inner and outer region of the survey area, we still find a uniform density of $\sim 100$ AGN per square degree that satisfy our selection criteria. Additionally, due to the work by Brand et al. (2006), the majority $(\sim 93 \%)$ of the sample also has an optical detection.

The mid and far-IR photometry requirement is unique to this work. Comparable studies required only one mid or far-IR detection or relied on stacking techniques and photometric upper limits to supplement, creating large uncertainties when generating AGN SEDs, particularly on the Wien side of the far-IR SED corresponding to dust emission (e.g. Mullaney et al. 2012; Stanley et al. 2015;
Lanzuisi et al. 2017). With the mid and far-IR requirement, we can better constrain dusty torus emission for powerful AGN and host star-forming galaxies.

This study is based on the 703 X-ray sources in the Boötes field that have intensive multiband data to fit their individual spectral energy distributions (see Table 1 for exact counts per band). Generating individual SEDs allows us to avoid the restrictions and uncertainties related to stacking and gives us the freedom to disentangle AGN and host galaxy radiation components for each respective source. Using SED3FIT (Berta et al. 2013), a multi-component SED fitting tool, we decompose each galaxy's emissions in the infrared spectrum and use the appropriate rest-frame, infrared luminosity integrated from $8 \mu \mathrm{m}$ to $1000 \mu \mathrm{m}$ as an indicator of host galaxy star formation rate. SED3FIT is based off of the da Cunha et al. (2008) MAGPHYs code and employs a combination of three galaxy radiation processes: stellar emission, warm and cold dust emission from star formation regions, and AGN emission. SED templates are fitted to measured fluxes first using the stellar and star forming components only, then AGN templates are varied to fill in photometric gaps and further reduce the $\chi^{2}$. We use the ten AGN templates provided with SED3FIT, which were selected to cover the wide range of AGN found in the full Fritz et al. (2006) library. These ten templates span Type 1, intermediate, and Type 2 AGN, with a variety of optical depths ranging from 0.1-6, as viewed face on or edge on. All ten of the templates have a fixed torus opening angle of $\Theta=100^{\circ}$, corresponding to an intrinsic covering factor of $75 \%$ (see Section 4.3 for details on covering factors). Each AGN template can be broken down into three components: dust scattering emission, dust thermal emission and nuclear accretion disk emission. The former two AGN components combined are attributed to the warm, dusty clumpy structure that surrounds the SMBH and accretion disk. See Figure 4 for two example spectral energy distributions generated from our sample (left: star formation dominant, right: AGN emission dominant).

To correct for contaminating AGN radiation, we subtract the dusty torus and accretion disk emission from 

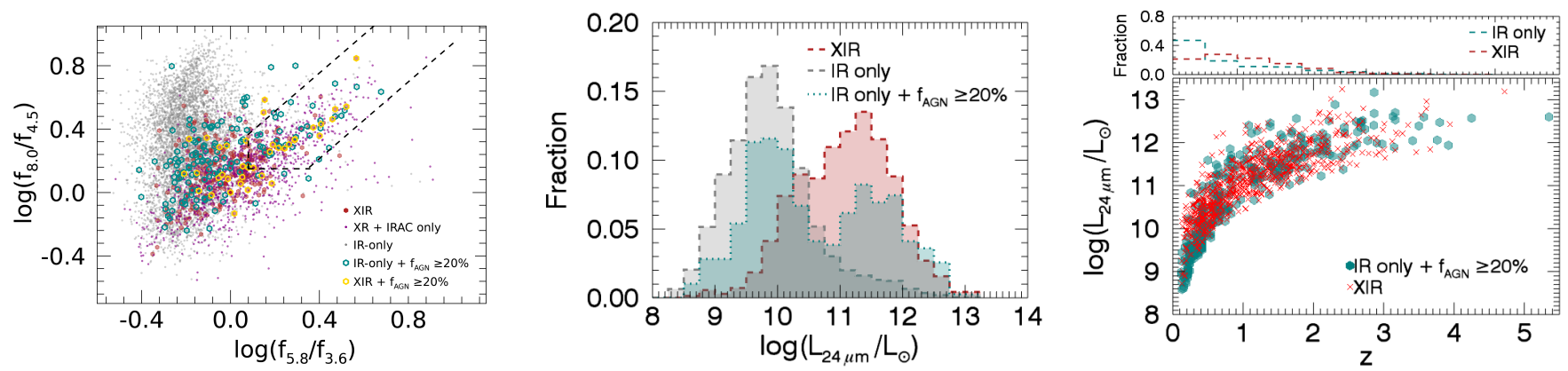

Figure 5. Left: IRAC color-color space used to identify luminous AGN in Donley et al. (2012). Grey points represent the IR-only sources with spec-zs, large red dots mark sources from our main sample of XIR sources, and purple points are additional X-ray sources with IRAC counterparts, but no mid or far-IR counterparts. The dashed black lines carve out the region belonging to luminous AGN, with minimal contamination from high redshift star-forming galaxies. We note that $15 \%$ of the luminous AGN in the IR only sample also have estimated AGN IR contributions $\geq 20 \%$ (teal circles), while the same is true for $25 \%$ of our XIR sample (gold circles). Generally, sources with AGN IR contributions $>20 \%$ are dispersed throughout this IRAC color space, indicating that SED decomposition does not lend itself to luminous AGN identification. Middle: $\mathrm{L}_{24 \mu m}$ distribution for the $703 \mathrm{X}$-ray detected sample (red), the 5k IR-only detected sample (grey), and the 389 IR-only sources with $\geq 20 \%$ of IR SED emissions coming from AGN processes (teal). Right: $\mathrm{L}_{24 \mu \mathrm{m}}$ vs. redshift distribution for both the 389 IR-only sample with significant IR AGN contribution (teal) and the X-ray detected sample (red). Top histogram represents the fraction of sources from each sample in redshift bins of size 0.5 .

the total SED of a source. The resulting infrared luminosity is attributed to star formation and is hereby represented as $\mathrm{L}_{\mathrm{IR}}^{\mathrm{SF}}$, while the subtracted infrared AGN luminosity is referred to as $\mathrm{L}_{\mathrm{IR}}^{\mathrm{AGN}}$; Figure 3 (top) shows our resulting population distribution of infrared luminosities attributed to star-formation processes. This procedure applies to $98 \%$ of our sample as 13 sources are not fitted with an IR AGN component by SED3FIT. The physical characteristics derived from this procedure will be available for all 703 sources on Vizier. ${ }^{3}$

Out of the remaining 2.6k XBoötes sources not used in our XIR sample, we also find 425 X-ray AGN with spectroscopic redshifts but no Spitzer MIPS and Herschel counterparts (marked as purple circles in Figure 2) with a similar X-ray and redshift distribution as our main sample - dubbed the non-IR sample (although some of these sources have IRAC detections; see next paragraph). We compare these non-IR AGN plus a sample of 6,583 IR-only galaxies with spectroscopic redshifts to our main sample in section 4 . The IR-only galaxies have both a MIPS $24 \mu \mathrm{m}$ and at least one Herschel far-IR detection, but no X-ray detection. For the non-IR AGN, we use the Herschel SPIRE $250 \mu \mathrm{m} 5 \sigma$ limiting flux in the deeper region of the HerMES survey as a generous upper limit on star formation luminosity. Out of the IR-only galaxy sample, $99 \%$ of sources have an optical counterpart and 91\% have an IRAC detection. We ran IR-only photometric data through SED3FIT and found only $72 \%(\sim 5 \mathrm{k})$ of the $\sim 6.6 \mathrm{k}$ galaxies are fitted with an AGN component.

For additional context, we briefly explore the additional two sample populations $(6.6 \mathrm{k}$ IR-only galaxies and 425 non-IR AGN) in IRAC color-color space in Figure 5 (left). Nearly $40 \%$ of the non-IR AGN (small purple dots) and $92 \%$ of the IR-only galaxies (grey points) have sufficient $(3 \sigma)$ detections in all four IRAC bands; the same is true for $46 \%$ of our main XIR AGN sample (large red dots). In the Donley et al. (2012) IRAC color criteria for identifying luminous AGN $\left(\mathrm{L}_{2-10 \mathrm{keV}} \geq 10^{44} \mathrm{ergs} \mathrm{s}^{-1}\right.$; wedge outlined by dashed black lines), 60 of 327 XIR sources with detections in all four IRAC bands are categorized as luminous

\footnotetext{
3 http://vizier.cfa.harvard.edu/
}

AGN with a median $\mathrm{L}_{2-10 \mathrm{keV}} \sim 5.6 \times 10^{43} \mathrm{ergs} \mathrm{s}^{-1}$; only 23 of the 58 XIR sources with $\mathrm{L}_{2-10 \mathrm{keV}} \geq 10^{44} \mathrm{ergs} \mathrm{s}^{-1}$ and IRAC detections are categorized as luminous AGN through the IRAC criteria, which is nearly equivalent to the X-ray luminous AGN recovery rate found in Donley et al. (2012) (38\%). This shows that, by using X-ray selection criteria, we're probing a larger population of the most powerful AGN. However, we must note that some powerful AGN are heavily obscured and therefore less Xray bright $(30-60 \%$, see Section 4.3$)$; we caution readers to consider this selection effect throughout this work.

In the same space, $7 \%$ of the non-IR AGN are categorized as luminous AGN with a median $\mathrm{L}_{2-10 \mathrm{keV}} \sim 2.3 \times 10^{44} \mathrm{ergs} \mathrm{s}^{-1}$ and a recovery rate of $32 \%$ for all X-ray luminous AGN in the non-IR sample; and out of the $5.6 \mathrm{k}$ IR only sources with spec-zs and sufficient IRAC detections, only $2 \%(\mathrm{~N}=128)$ of sources are deemed luminous AGN (but members of this sample do not have any bona fied X-ray detections, so we cannot determine the recovery rate).

\section{RESULTS AND DISCUSSION}

\subsection{Average $L_{I R}^{S F}$ vs. Average $L_{\mathrm{AGN}}$}

We translate X-ray flux to bolometric AGN luminosity, $\mathrm{L}_{\mathrm{AGN}}$, using the equation in Rosario et al. (2012) (R12 hereafter) derived from Maiolino et al. (2007) and Netzer \& Trakhtenbrot (2007) for spectroscopically confirmed Type 1 (unobscured) AGN:

$$
\log \mathrm{L}_{\mathrm{AGN}}=\frac{\log \mathrm{L}_{\mathrm{X}}-11.78}{0.721}+0.845
$$

where $\mathrm{L}_{\mathrm{X}}$ is the $2-10 \mathrm{keV}$ band $\mathrm{X}$-ray luminosity. We average infrared contribution from star forming processes in bins of $\mathrm{L}_{\mathrm{AGN}}$, with respect to each redshift interval, and do the same separately for the additional $425 \mathrm{X}$ ray sources with spectroscopic redshifts but no IR counterparts. We show these results in Figure 6(left); the dashed line represents the relationship found in Netzer (2009) (N09, hereafter) for local, low luminosity AGNdominated systems where $\mathrm{L}_{\mathrm{AGN}}$ is much larger than $\mathrm{L}_{\mathrm{IR}}$. Nearly $50 \%$ of X-ray only detected sources fall into the AGN-dominated section, compared to only $\sim 5 \%$ of individual X-ray and IR detected sources, substantiating the 

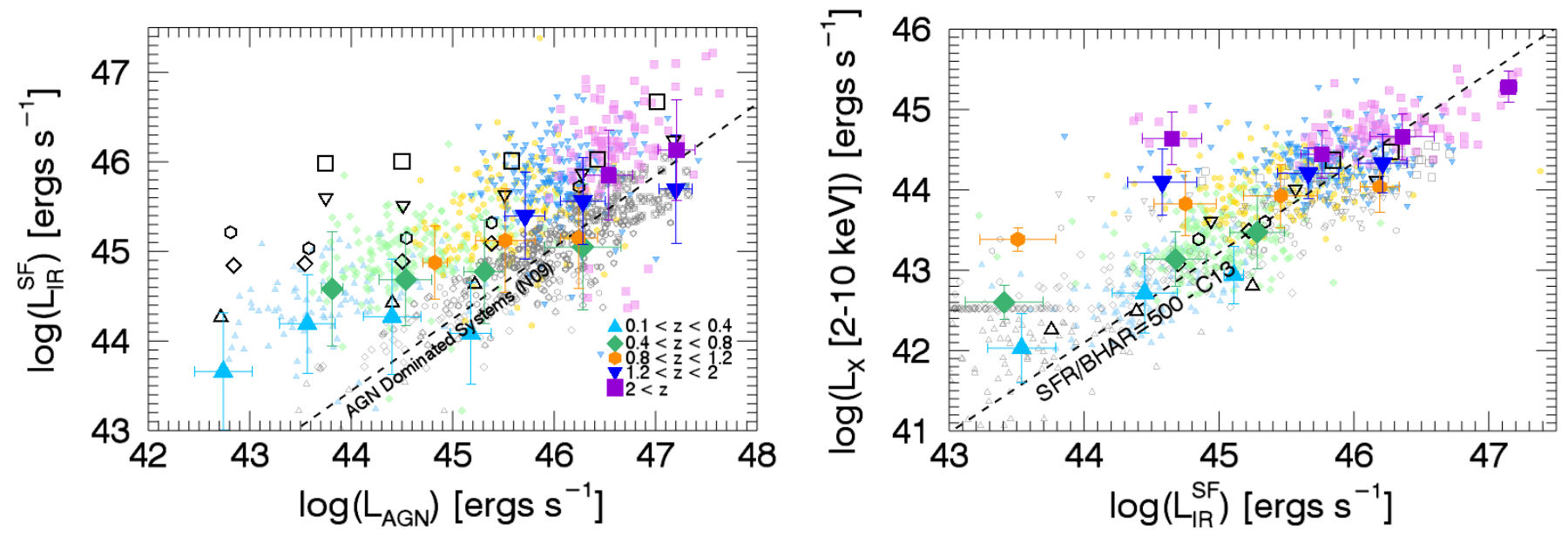

Figure 6. Left: Distribution of AGN bolometric luminosity $\left(\propto \mathrm{L}_{\mathrm{X}}\right)$ versus $\mathrm{L}_{\mathrm{IR}}^{\mathrm{SF}}$. The lighter, smaller points are individual AGN. The small, empty gray symbols are X-ray AGN with $\mathrm{L}_{\mathrm{IR}}^{\mathrm{SF}}$ upper limits determined by the HerMES Herschel SPIRE $250 \mu \mathrm{m}$ flux limit. The larger, bolder, filled in points are average $\log \left(\mathrm{L}_{\mathrm{IR}}^{\mathrm{SF}}\right)$ in bins of $\log \left(\mathrm{L}_{\mathrm{AGN}}\right)$ showing both the IR detected (colorful) and IR non-detected (empty grey) X-ray sources. Error bars represent the $1 \sigma$ dispersion of each bin. Note the star forming luminosity for the most powerful AGN in the $0.4<z<0.8$ z-bin lies directly under the corresponding average star forming luminosity for the most powerful AGN in the $0.8<z<1.2$ z-bin. The black dashed line represents the relationship found in N09 where objects below the line have infrared luminosities dominated by AGN activity. Black empty symbols are results from Lanzuisi et al. (2017). Right: Average $\log \left(\mathrm{L}_{\mathrm{X}}\right)$ in bins of $\log \left(\mathrm{L}_{\mathrm{IR}}^{\mathrm{SF}}\right)$ compared to results from C13 (Chen et al. 2013). The C13 sample is represented by the black empty shapes. The dashed line is the constant proportional relationship between star formation rate (SFR) and black hole accretion rate (BHAR) found in C13. Colors, symbols and error bars are calculated in the same fashion as in the left figure, where the empty gray points denote the IR only detected sources with an estimated IR AGN fraction $\geq 20 \%$ with X-ray upper limits defined by the XBoötes survey flux limit.

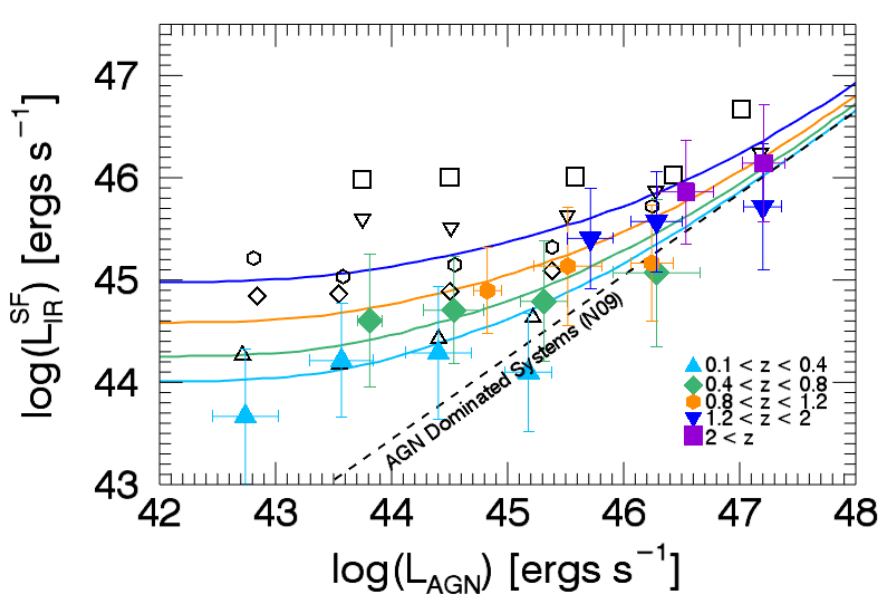

Figure 7. Distribution of AGN bolometric luminosity versus $\mathrm{L}_{\mathrm{IR}}^{\mathrm{SF}}$ with the black dashed line as defined in Figure 6 (left). The solid lines have colors corresponding to redshift ranges and are the extrapolated trends from the Hickox et al. (2014) simple model incorporating short-term AGN variability, long-term evolving star formation rates and a universal constant of proportion between SFRs and black hole accretion rates.

selection of AGN embedded within star-forming galaxies in this analysis and demonstrating the dominance of star-formation driven modes in IR luminosities of $\mathrm{Her}$ schel detected dusty galaxies. This trend is corroborated in several recent works using IR-bright X-ray selected AGN (e.g. R12, L17, Dai et al. 2017), indicating that the power law correlation from N09 is valid when extended to higher luminosities and high-z AGN.

Our low-z $(z \lesssim 1)$ sample successfully reflects those of other published results with low luminosity AGN $\left(\mathrm{L}_{\mathrm{AGN}}<10^{45} \mathrm{erg} \mathrm{s}^{-1}\right)$ showing a flat or uncorrelated relationship between AGN activity and star formation. The higher luminosity AGN in the low-z bins appear to trend in a more positive linear fashion that approaches the N09 relationship. The stronger, positive relationship is most noticeable in the $0.4<z<0.8$ bin where the most powerful AGN, while few in number $(\mathrm{N}=6)$, are embedded in star-forming galaxies nearly just as bursty as the brightest AGN in the $0.8<z<1.2$ bin. These results also appear in L17 and R12, but conflict with the flat, nonexistent relationships found in Stanley et al. (2015) and Dai et al. (2017).

Hickox et al. (2014) and Volonteri et al. (2015) developed models that match similar observational results as seen in L17, R12, Chen et al. (2013) and Azadi et al. (2015). In Figure 7, we overlay the Hickox et al. (2014) model curves and see general agreement with the results for our $z \sim 1$ less powerful active galaxies $\left(\mathrm{L}_{\mathrm{AGN}}<10^{45} \mathrm{ergs} \mathrm{s}^{-1}\right)$, but the model over estimates star forming luminosity for the more powerful AGN $\left(\mathrm{L}_{\mathrm{AGN}}>10^{45} \mathrm{ergs} \mathrm{s}^{-1}\right)$ in each redshift range. To create the model, Hickox et al. (2014) generated a sample of galaxies (up to $z=2$ ) in which all star-forming galaxies host an AGN during their lifetime, and then incorporated a constant of proportion between SFR and black hole accretion rate over long time scales $(\log (\mathrm{SFR} / \mathrm{BHAR})=3.6$ (Chen et al. 2013; Dai et al. 2017)) and assigned short time scale variabilities in AGN accretion processes (and therefore, luminosity). Generally, the model successfully produces the observed findings when averaging star formation activity in bins of AGN activity, along with the trends observed in literature when averaging AGN activity in bins of star-formation activity, as analyzed in the following section.

\subsection{Average $L_{X}$ vs. Average $L_{\mathrm{IR}}^{\mathrm{SF}}$}

Recent simulations and observations reveal that AGN accretion (and therefore luminosity) can be highly variable on short timescales - e.g. on the order of 1-2 magni- 
tudes within 0.1-1 Myr (e.g. Di Matteo et al. 2005; Hickox et al. 2014) - whereas star formation processes change at a slower rate over longer timescales. To uncover the relationship between AGN processes and host galaxy star formation rates, it might be more appropriate to average AGN activity (the more rapidly changing variable) based on $\mathrm{L}_{\mathrm{IR}}^{\mathrm{SF}}$ (the more stable variable).

Following the analysis in L17 and Chen et al. (2013) (C13, hereafter), we reversed data dependency by averaging $\log \left(\mathrm{L}_{\mathrm{X}}\right)$ in bins of $\log \left(\mathrm{L}_{\mathrm{IR}}^{\mathrm{SF}}\right)$. We include $389 \mathrm{IR}$ only sources with an AGN IR contribution that is $\geq 20 \%$ of the total IR SED. These IR-only sources have both a MIPS $24 \mu \mathrm{m}$ and at least one Herschel far-IR detection, but no X-ray detection (see Figure 5 for $24 \mu \mathrm{m}$ population distribution). We take the ratio of IR AGN luminosity to total IR luminosity from the resulting SED and place a cut at $\geq 20 \%$ to capture the sources with the highest likelihood of hosting an AGN (Ciesla et al. 2015). For these objects, we use the XBoötes survey flux limit as an upper limit for X-ray luminosity. Results are shown in Figure 6 (right) with L17 results overlaid. Error bars represent the $1 \sigma$ dispersion of the mean X-ray luminosity in each respective bin. The dashed line represents the constant ratio between black hole accretion rate (BHAR, proportional to X-ray luminosity) and star formation rate found in $\mathrm{C} 13$ for 34 X-ray detected AGN at $z=0.25-0.8$.

We find our results to be in good agreement with the $\mathrm{C} 13 \mathrm{SFR} / \mathrm{BHAR}$ ratio. The low z-bins $(z \lesssim 1)$ have the strongest positive slope between the same $\mathrm{L}_{\mathrm{IR}}^{\mathrm{SF}}$ intervals studied in C13, which is expected as C13 analyzed data from the same Boötes Chandra, Herschel and Spitzer observations used in this paper. While AGN still hover near the SFR/BHAR ratio in the earlier $z>0.8$ Universe, there is no significantly strong upward trend as $\mathrm{L}_{\mathrm{IR}}^{\mathrm{SF}}$ increases for any z-bin, and the nearly $\sim 0.5$ dex increase exhibited within the $z>2$ sample for the highest range of star formation activity has a very small sample size and is therefore unreliable.

Note that these observations are limited to the depths of the $24 \mu \mathrm{m}$ survey; an object at $z \sim 1$ with a $24 \mu \mathrm{m}$ luminosity of $L_{24 \mu \mathrm{m}}=10^{44} \mathrm{ergs} \mathrm{s}^{-1}$ is pushing the survey observational limits and might be undetected. This means that the weakest star formation bins in this analysis may be lacking contributions from some fainter, intermediate redshift galaxies and AGN. Conversely, some powerful AGN are heavily obscured by high column densities of dust and gas. In fact, studies have shown that $90 \%$ galaxies with high $24 \mu \mathrm{m}$ to optical flux ratios have IR and X-ray signatures indicating the presence of heavily obscured AGN (Fiore et al. 2008; Treister et al. 2009). These AGN are expected to have intrinsic X-ray luminosities in excess of $10^{44} \operatorname{ergss}^{-1}$, at $z \sim 1-2$, which could drive the more star-forming $\mathrm{L}_{\mathrm{IR}}^{\mathrm{SF}}$ bins further upward and into stronger agreement with the C13 trend.

The observed differences in correlation between the two averaging methods are likely due to the inherent rate of variation between the two physical processes, with star formation being the more stable measurement and AGN accretion being the more variable measurement. These differences in correlation methods were also confirmed by Dai et al. (2017) for similar samples of X-ray selected AGN. Lapi et al. (2014) found similar results when exploring the observational phenomena of the coevolutionary relationship between AGN and host galaxies at high redshifts $(z \gtrsim 1.5)$ using a semi analytical model. Combining observational data on AGN in starforming galaxies with high- $z$ AGN luminosity functions and host-galaxy stellar luminosity functions, the model shows galaxy SFRs that remain relatively constant over a long period of time then suddenly undergo a rapid decrease in star formation when the SMBH is triggered into an active phase. The model also predicts that as the supermassive black hole grows, a fraction of the cold interstellar gas and dust within the spiral arms of a galaxy is drawn towards the nucleus to help form and grow the dusty torus. The AGN will feed off this reservoir and the most powerful AGN will have feedback processes that strip away some of the remaining cold gas and dust, further suppressing star formation processes and eventually slowing its own growth as well. Observations at various epochs within the model easily reproduce both of the trends shown in Figure 6 and, when combined with the publications and findings discussed in section 4.1 , indicate that a more detailed study on the relationship between short term AGN variability and host galaxy cold gas and dust properties is necessary to arrive at any definitive conclusions.

\subsection{Dust Covering Factors}

We can determine how dust obscured an accreting $\mathrm{SMBH}$ is by assessing the relationship between how much high energy radiation from accretion disk processes is observed (which therefore escapes the dusty torus), versus how much radiation is detected from the dusty torus itself. A commonly used dust covering factor (CF) proxy is the ratio of dusty torus emission, $\mathrm{L}_{\text {Tor }}$ (which dominates in the mid to far-IR), to bolometric AGN luminosity, LAGN (e.g. Maiolino et al. 2007; Treister et al. 2008; Rowan-Robinson et al. 2009). To compute the dust covering factor for our sample, we use the bolometric AGN luminosities derived from Equation (2), and derive $\mathrm{L}_{\text {Tor }}$ from the dusty torus components in each source's respective AGN SED (i.e. we remove the infrared emission originating solely from the accretion disk from each AGN SED template for each source and keep only the dusty torus emission components). We caution that systematics from the fixed covering factor $(75 \%)$ in the AGN SEDs may produce biased estimates of dusty torus emission in this analysis (see Section 3).

We note that this proxy $\left(\mathrm{CF}=\mathrm{L}_{\mathrm{Tor}} / \mathrm{L}_{\mathrm{AGN}}\right)$ is used under the assumption that accretion disk emission and the resulting dusty torus emission are generally isotropic. However, the work of Stalevski et al. (2016) shows that, when considering the anisotropy of these emission processes for Type 1 AGN with $\mathrm{L}_{\mathrm{AGN}} \sim 10^{45} \mathrm{ergs} \mathrm{s}^{-1}$, this proxy can underestimate intrinsically low covering factors and overestimate high covering factors, while for Type 2 AGN of similar luminosities, this proxy always underestimates the true covering factor. We assess the impact of this assumption on our results at the end of this section.

The average dust covering factor decreases with an increase in AGN activity for our X-ray detected AGN sample (Figure 8, left). This trend correlates nicely with the luminosity-dependent AGN unified model where dust covering factor is anti-correlated with bolometric lumi- 

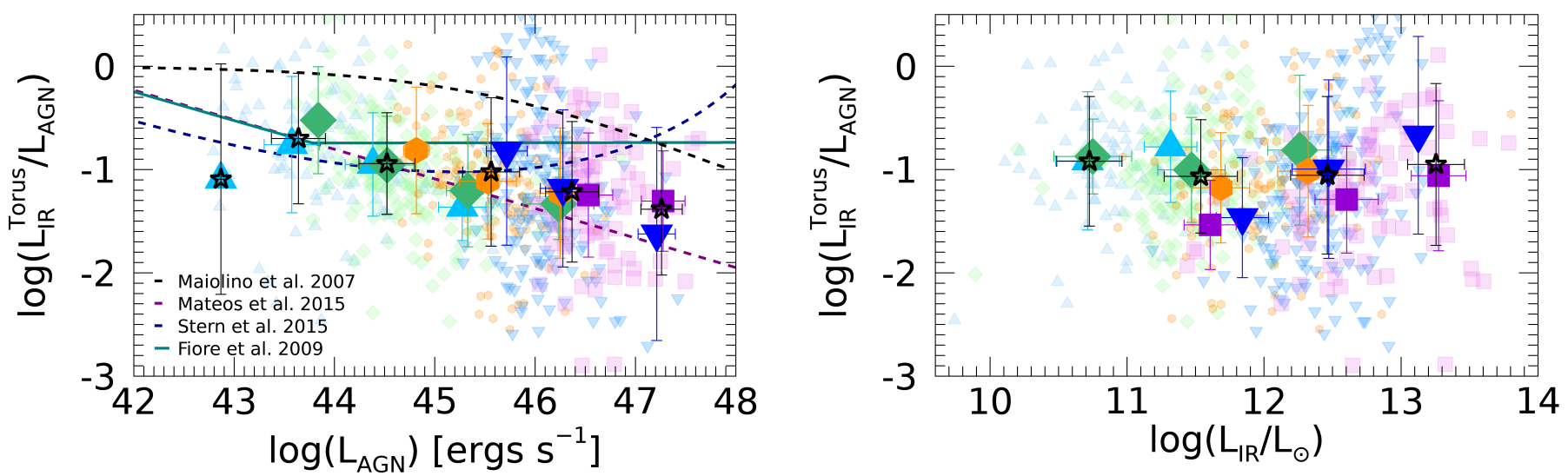

Figure 8. Left: Covering factor versus bolometric AGN luminosity. Averages for the X-ray detected sample are computed in bins of $\mathrm{L}_{\mathrm{AGN}}$ and in respective redshift ranges. We also computed averages for the entire sample, irrespective of redshift range, as indicated by the empty black stars. The black dashed line represents the fraction of obscured AGN as a function of bolometric AGN luminosity found by Maiolino et al. (2007). The purple dashed line, navy dashed line, and turquoise solid line correspond to mid-IR/L $\mathrm{L}_{\mathrm{AGN}}$ fractions found by translating the X-ray-to-6 $\mu \mathrm{m}$ relationships derived in Mateos et al. (2015), Stern (2015), and Fiore et al. (2009), respectively. Right: Covering factor versus total infrared luminosity. Averages, colors and symbols are derived in the same fashion as the figure to the left.

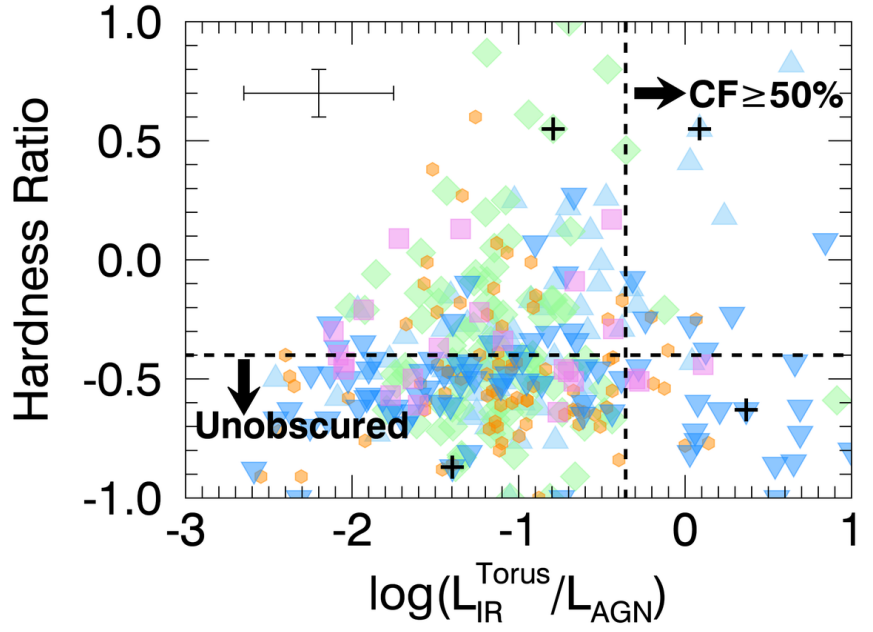

Figure 9. Covering factor versus hardness ratio for the 330 XIR AGN with sufficient x-ray counts to determine hardness ratios. Colors and shapes are the same as those in Figure 6. Average error bars are presented in the top left corner. To the right of the black dashed vertical line lies AGN with covering factors greater than $50 \%$. Below the black dashed horizontal line lies AGN with hardness ratios indicative of unobscured cores. SEDs for sources marked with crosses are in the appendix, providing examples of some of the more extreme and contradictory AGN in this sample.

nosity, also known as the receding torus model (Lawrence 1991). Taking the model implications a step further, it follows that the average covering factor within a sample of AGN corresponds directly to the fraction of Type 2 (obscured) AGN. In this work, we find an average $\mathrm{CF}$ of $33 \%$ for the X-ray detected AGN. This average $\mathrm{CF}$ is similar but slightly lower than those found in literature: Rowan-Robinson et al. (2009) used Chandra and/or Spitzer data to determine CFs for 658 AGN and found an average dust covering factor of $40 \%$; Mateos et al. (2015) determines a spectroscopically confirmed Type 2 fraction of $43 \%$ on a sample of 250 X-ray selected AGN with dust covering factors ranging from 20-50\% when averaged in bins of X-ray luminosity; Lanzuisi et al. (2009) found a higher Type 2 fraction at $55 \%$ of mid-IR bright X-ray selected AGN, and Hickox et al. (2007) selected IR-AGN in the same field as this study and used spectroscopic and optical to mid-IR color distributions to determine a Type 2 fraction of $43 \%$. The observed luminosity-dependence agrees most with the trend found in Mateos et al. (2015) (shown as the purple dashed line in Figure 8, left), who also used multi-component SEDs to determine the AGN contribution to mid-IR luminosity. A newer study by Mateos et al. (2017) investigated the lack of one to one correlation between Type 2 fraction and average covering factor for their complete sample of optically classified X-ray AGN. They identify a missing population of X-ray obscured AGN and, when the high covering factors of these obscured AGN are accounted for, the population $\mathrm{CF}$ average grows to nearly $60 \%$ with a less significant luminosity dependence relationship. It is possible that the CFs of heavily obscured AGN in the Boötes region would effectively raise the average $\mathrm{CF}$ across all redshift ranges and AGN luminosities to a similar value, but that analysis it out of scope for this work.

In Figure 8, right, we find an overall flat relationship between total infrared luminosity and covering factors for the X-ray selected sample, hovering at an average of $\sim 10 \%$ across all luminosities. While there appears to be some positive relationship for all redshift bins $z>0.4$ starting at $\log \left(\mathrm{L}_{\mathrm{IR}} / \mathrm{L}_{\odot}\right) \approx 11.5$, the sample dispersion is large, spanning $\pm \sim 50 \%$ (or more) for each average data point within each redshift bin. Therefore, any observed positive correlation is weak and would require further investigation for verification.

We also recover trends that challenge the inclinationbased unified model: there is no clear bimodal distribution for covering factors in the XIR AGN population; instead we see a distribution of covering factors that cover the entire possible range at significant percentages. To investigate, we further restrict our sample to the 330 XIR AGN with sufficient X-ray counts to determine hardness ratios (HRs; i.e. $\mathrm{H}-\mathrm{S} / \mathrm{H}+\mathrm{S}$, an indicator of AGN obscuration; e.g. Green et al. (2004)) and find the majority $(\sim 57 \%)$ are unobscured with corresponding HRs $\lesssim-0.5$ and an overall wide spread in covering factors averaging at $35 \% \pm 1.03 \%$ (see Figure 9). Concentrating only on the 187 XIR AGN with unobscured HRs, we find 11\% have 

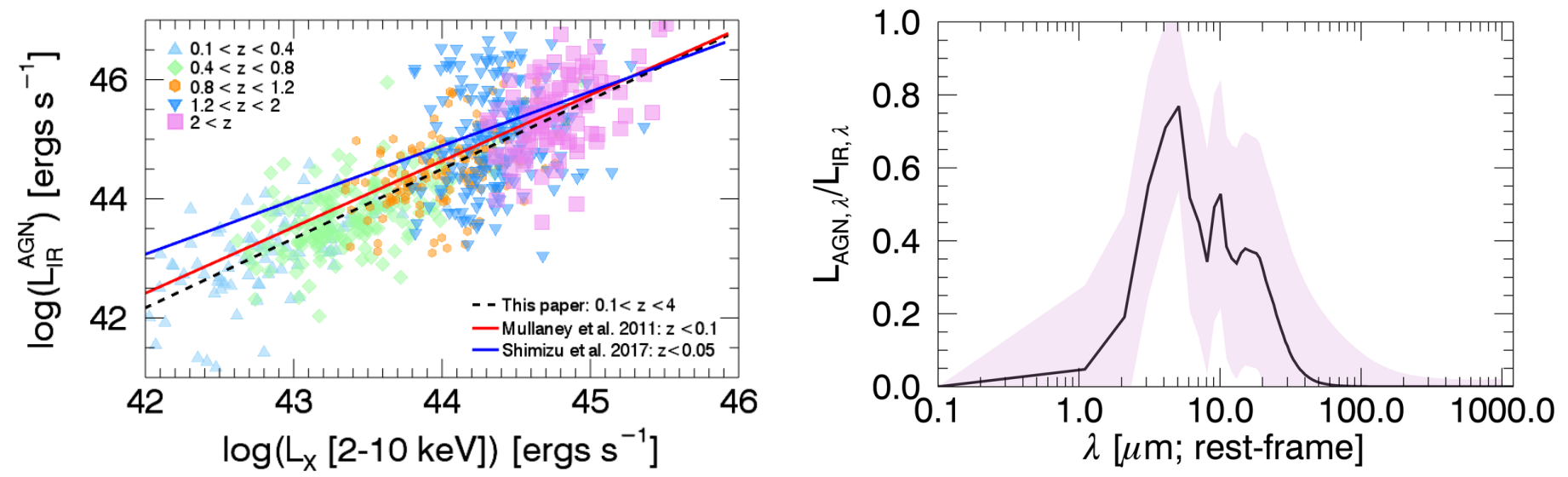

Figure 10. Left: Infrared AGN luminosity as a function of $\mathrm{L}_{\mathrm{X}}$ for the X-ray AGN. The black dashed line represents the linear relationship found in log space between the two AGN luminosities. The red and blue lines represent the relationship determined from generated average SEDs for local AGN by Mullaney et al. (2011) and Shimizu et al. (2017), respectively. Right: Composite $f_{\text {AGN }}$ as a function of rest-frame wavelength using all $703 \mathrm{X}$-ray selected AGN. The black line is the median value at all wavelengths in bins of $\Delta \lambda=1 \mu \mathrm{m}$ and the $1 \sigma$ scatter for each $\Delta \lambda$ is indicated by the shaded pink region.

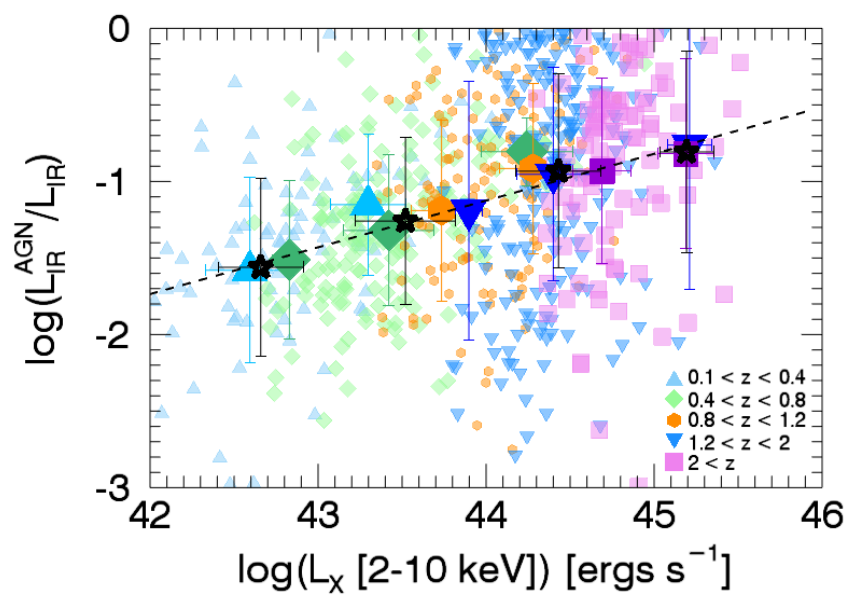

Figure 11. AGN fractions for the X-ray detected sample as a function of X-ray luminosity. Averages are computed in bins of $\mathrm{L}_{\mathrm{X}}$, respective of redshift range, with error bars representing the $1 \sigma$ dispersion of the mean. Black empty stars represent the averages across X-ray luminosity, regardless of age in the universe.

$\mathrm{CFs} \gtrsim 50 \%$, indicating that a defining $\mathrm{CF}$ cut off limit between Type 1 and Type 2 AGN based on X-ray absorption is nonexistent. Mateos et al. (2016) found similar results using 227 spectroscopically confirmed and categorized X-ray AGN; while the different types of AGN had clearly different $\mathrm{CF}$ distributions (with type 2(1) peaking at high(low) covering factors) there was still a very strong overlap in CF distributions; roughly $20 \%$ of Type 1 AGN had CFs $>0.5$ and $40 \%$ of Type 2 AGN had CFs $<0.5$. Merloni et al. (2014) used optical photometry and/or spectra paired with hard X-ray data for $\sim 1300$ AGN and found $31 \%$ of the entire sample sits in a similar contradictory region where optical signatures point towards an unobscured nucleus while X-ray data indicates considerable gas and dust absorption, or vice versa with optical evidence for an obscured nuclear region and no absorption of soft X-rays. This work and the aforementioned suggest that Type 1 and Type 2 AGN may not be observationally distinct due to the line-of-sight inclination of the dusty torus but instead due to other physical accretion related mechanisms.

Recently, Ricci et al. (2017) showed that the relation- ship between AGN luminosity and covering factor flattens out when dividing X-ray AGN into separate bins of Eddington ratios $\left(\lambda_{E}\right.$; mass-normalized black hole accretion rate), indicating that the AGN line-of-sight obscuration is not the universal driver of covering factor distributions. Instead, $\lambda_{E}$ and $\mathrm{CF}$ maintain a steady positive correlation up until the sublimating Eddington limit for dusty gas particles, in which the CF sharply declines. These results point towards strength in radiation pressure from accretion activities being the main regulator of observed obscuration fractions, and that Type 1 and Type 2 AGN are actually physically different objects (as categorized by $\lambda_{E}$ ) that could be better unified within the context of black hole growth over time. Exploration of this relationship is out of scope for this analysis; we refer readers to Beckmann et al. (2009); Winter et al. (2009); Ezhikode et al. (2016); Lusso et al. (2012); Lawrence \& Elvis (2010); Mateos et al. (2017) for further discussions that precede the Ricci et al. (2017) results.

We explored how the Stalevski et al. (2016) equation and coefficients (Equation 8 and Table 1, inside) for correcting isotropically-assumed dust covering factors affect our results by first identifying Type 1 and Type 2 AGN using the inclination angles used in the SED fitting procedure. The AGN SED fitting model used in this paper include two possible nuclear line of sight angles: 0 degrees (face-on aka Type 1 unobscured nucleus) or 90 degrees (edge-on aka Type 2 nucleus viewed through the disk). Based on this criteria, $61 \%$ of our XIR sources are categorized as Type 1 AGN and the remainder are categorized as Type $2 \mathrm{AGN}$, which is consistent with the average $\mathrm{CF}$ derived earlier in this section. Interestingly, the majority of Type 2 AGN in this sample $(77 \%)$ have covering factors below $\leq 10 \%$, while Type 1 AGN exhibit no general CF preference. Both AGN types have median AGN luminosities of $\mathrm{L}_{\mathrm{AGN}} \sim 3 \times 10^{45} \mathrm{ergs} \mathrm{s}^{-1}$.

We applied each set of coefficients corresponding to the three reported example optical depths $\left(\tau_{9.7 \mu \mathrm{m}}=3,5,10\right.$ in Stalevski et al. (2016)) to the respective AGN types. The overall effect is strongest for AGN (of both types) with originally estimated CFs less than $20 \%$, which is nearly three quarters of the $703 \mathrm{AGN}$; for each set of coefficients, dust covering factors were increased to $\geq 20 \%$, 

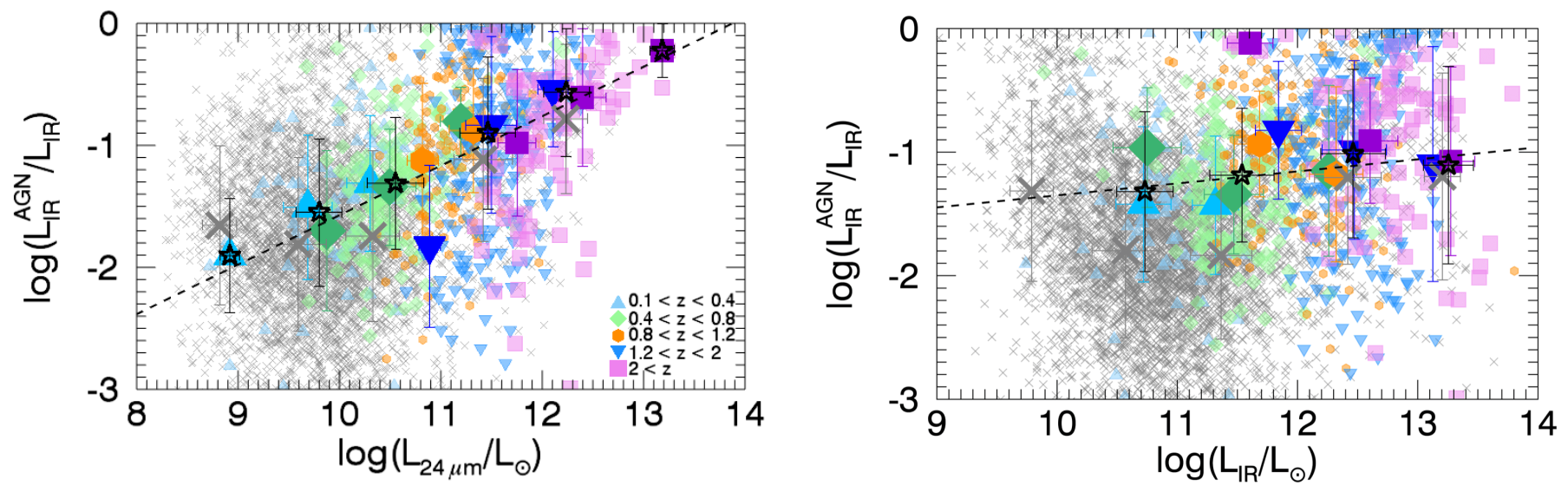

Figure 12. These two figures represent the ratio of infrared AGN luminosity to host galaxy total infrared luminosity (i.e. AGN fraction) as a function of host galaxy luminosities. Colors and symbols are the same as in Figure 8 , with grey x's denoting the individual $\sim 6 \mathrm{k}$ IR-only galaxies as defined in Section 3 and their averages in luminosity buckets of size 1 dex are represented as large grey x's. Left: The logarithm of the AGN fraction as a function of $24 \mu \mathrm{m}$ luminosity. The XIR sample shows a clear, but weak, correlation between $24 \mu \mathrm{m}$ luminosity and AGN fractions. Right: The logarithm of the AGN fraction as a function of total infrared luminosity, following the same legend as in the figure on the left. There appears to be no clear relationship between total IR luminosity and AGN fraction, indicating a need for individual IR SED decomposition when estimating AGN fractions across IR luminosity space.

due to the lower limits assumed in Stalevski et al. (2016), with the average individual differences being $+33 \%$ to the respective CFs. This effectively flattens out any trends seen in Figure 8, where the original average CF of $33 \%$ is now a corrected average CF of $49 \%$. It is worth noting that these equations were originally derived for a luminous AGN with $\mathrm{L}_{\mathrm{AGN}} \sim 10^{45}$ ergs s$^{-1}$; the third of our sample at lower AGN luminosities sees an average CF correction of $\sim+25 \%$, while the remaining more powerful population has a ten percent higher average $\mathrm{CF}$ correction than that of the low luminosity AGN. Thus, due to the the underlying assumptions in covering factors and the wide range in AGN luminosities probed in this work, we are unfortunately limited from interpreting any further.

\subsection{AGN Contribution in the Infrared}

The wide infrared coverage in the Boötes region when paired with the multicomponent SED fitting model SED3FIT is advantageous in effectively constraining intrinsic infrared AGN luminosities across a broad redshift range. This is useful to avoid situations of overestimating host galaxy properties (e.g. star formation rates) in cases with little IR photometry and/or possible indications of AGN activity. In the following, we explore the extracted infrared AGN luminosities in the $8-1000 \mu \mathrm{m}$ range $\left(\mathrm{L}_{\mathrm{AGN}}^{\mathrm{IR}}\right.$, hereafter) as a function of $\mathrm{L}_{\mathrm{X}}$, as well as the fraction of total infrared luminosity attributed to AGN emissions ( $\mathrm{L}_{\mathrm{AGN}}^{\mathrm{IR}} / \mathrm{L}_{\mathrm{IR}}$ or $f_{\mathrm{AGN}}$, hereafter) as a function of $\mathrm{L}_{\mathrm{X}}, \mathrm{L}_{\mathrm{IR}}$, and $\mathrm{L}_{24 \mu \mathrm{m}}$.

There is a strong correlation between X-ray activity and total infrared AGN luminosity within our X-ray detected sample. This relationship is similar to the driving trend determined in Mullaney et al. (2011), even though a large portion of our sample contains galaxies with low AGN fractions $\left(f_{\mathrm{AGN}}<10 \%\right)$ out to high redshifts. Mullaney et al. (2011) modeled intrinsic infrared AGN SEDs for only 11 local $(z<0.1)$ AGN with polycyclic aromatic hydrocarbon emission lines indicative of IR luminosities dominated by AGN $\left(f_{\mathrm{AGN}}>90 \%\right)$. As seen in Figure 10 (left), we derive a nearly equivalent relationship for AGN spanning a much larger redshift and AGN fraction range, suggesting that this relationship is universal. The black dashed line denotes our sample relationship, where

$$
\begin{aligned}
\log \left(\frac{\mathrm{L}_{\mathrm{IR}}^{\mathrm{AGN}}}{10^{43} \mathrm{erg} \mathrm{s}^{-1}}\right) & =(0.33 \pm 0.06) \\
& +(1.16 \pm 0.05) \log \left(\frac{\mathrm{L}_{\mathrm{X}}}{10^{43} \mathrm{erg} \mathrm{s}^{-1}}\right)
\end{aligned}
$$

with a strong, positive correlation coefficient of 0.78 . The red line denotes the slope found in Mullaney et al. (2011) $(1.11 \pm 0.07)$ and the blue line denotes the slightly weaker relationship found by Shimizu et al. (2017) (0.91 \pm 0.06$)$ who analyzed a sample of 313 local X-ray selected AGN with Herschel and WISE detections; additionally, Kirkpatrick et al. (2017) found a more extreme relationship (3.76 \pm 0.08 , not plotted) for $53 z \sim 1-2$ composite galaxies in the GOODS-S field with Spitzer and Herschel detections. Our work provides the first $0.1<z<4$ pure AGN infrared SED relationship estimated using a statistically significant population size, providing future studies the ability to estimate the total infrared emission of a high-z AGN using only X-ray data. A deeper X-ray study with a similar amount multiwavelength IR data and de-absorbed X-ray luminosities would be needed to confirm this relationship is complete to lower luminosity $\mathrm{X}$-ray AGN at $z>0.1$.

The median infrared AGN contribution across all sources is $8-30 \%$, indicating that roughly $70-90 \%$ of infrared light from this set of galaxies is coming from star formation processes. When restricting our sample to the 337 ULIRGs $\left(\mathrm{L}_{\mathrm{IR}}>10^{12} \mathrm{~L}_{\odot}\right)$, we find a median $f_{\text {AGN }}=13 \%$, similar to the fraction found in Nardini et al. (2008) for local ULIRGs. Looking at the median composite, rest-frame $f_{\text {AGN }}$ as a function of wavelength in Figure 10 (right), we find that AGN contribution heavily affects the mid-IR, with a maximum of nearly $80 \%$ at 5-6 $\mu \mathrm{m}$. While the impact of AGN contribution trails off at wavelengths greater than $\sim 30 \mu \mathrm{m}$ in Figure 10 (similar to other results, e.g. Kirkpatrick et al. (2012); Mullaney et al. (2011)), the $f_{\text {AGN }}$ sample distribution is broad at 
each wavelength with an average scatter of $\pm 20-30 \%$, implying that multi-component SED analysis is crucial in accurately determining the true AGN contribution for individual sources, particularly for cases without X-ray observations to constrain $\mathrm{L}_{\mathrm{AGN}}^{\mathrm{IR}}$.

On average, $f_{\text {AGN }}$ increases with increasing $\mathrm{X}$-ray and $24 \mu \mathrm{m}$ luminosity, but not with total infrared luminosity (see Figure 11 and Figure 12). The latter tells us that any trends found with $\mathrm{L}_{\mathrm{AGN}}^{\mathrm{IR}}$ are not driven simply by the host galaxy's luminosity; or, in other words, a broad range of infrared AGN fractions can be found embedded in variously luminous galaxies. As expected, for the IRonly galaxies (represented by grey x's) we see fairly low AGN fractions at low and average luminosities; yet, at higher luminosities, the IR-only galaxies and XIR AGN appear similar across the $\log \left(f_{\mathrm{AGN}}\right)-\log \left(\mathrm{L}_{24 \mu m}\right)$ relationship (possibly due to incomplete sample selection effects and/or SED modeling degeneracies for SMGs high redshifts, e.g. da Cunha et al. (2015)). The 128 IRonly galaxies with IRAC colors indicative of embedded luminous AGN (not highlighted; see Section 3 for sample definition) span a similar range of $24 \mu \mathrm{m}$ luminosities and follow nearly exactly the same trends as the XIR sample. We also considered the relationship between $f_{\mathrm{AGN}}$ and host galaxy stellar mass; again, we find a flat, nonexistent correlation. We note that this sample occupies a host galaxy stellar mass distribution similar to those found in literature for AGN host galaxies, with a mean stellar mass of $\log \left(\mathrm{M}_{*}\right)=10.83 \pm 0.58$ (e.g. Hickox et al. 2009; Xue et al. 2010).

We determine a clear but weak relationship in $\log \left(f_{\mathrm{AGN}}\right)-\log \left(\mathrm{L}_{24 \mu m}\right)$ and in $\log \left(f_{\mathrm{AGN}}\right)-\log \left(\mathrm{L}_{\mathrm{X}}\right)$, both with slopes $\approx 0.11$. Both correlations have large intrinsic scatters and weak correlation coefficients at $\sim \pm 60 \%$ and $\sim 0.36$, respectively. Ciesla et al. (2015) shows that $f_{\text {AGN }}$ predictions below $20 \%$ are accompanied with large uncertainties and therefore should be disregarded; these uncertainties vary across AGN types and it is unclear how they might vary across AGN luminosities. To investigate whether there is a stronger relationship present in our more certain $f_{\text {AGN }}$ calculations, we restrict our sample to $f_{\mathrm{AGN}} \geq 20 \%$, which is about $28 \%$ of the entire sample with an average $\log \left(\mathrm{L}_{24 \mu \mathrm{m}}\right)=11.56 \pm 0.66$ and $\log \left(\mathrm{L}_{\mathrm{X}}\right)=44.30 \pm 0.54$. Instead, we find an even weaker slope at $\sim 0.06$ with a correlation coefficient of $\sim 0.12$ for both sample populations, and again a large range of values. These results directly indicate a need for individual SED decomposition to infer the fraction of infrared output attributed by an AGN.

\section{SUMMARY AND CONCLUSIONS}

We explored the relationship between AGN activity and host galaxy dust properties across the tail end of peak AGN and galaxy growth in the Universe (redshifts $0.2<z<5$ ) using Chandra, Herschel, Spitzer and NOAO Telescope observations in the Boötes field. We successfully disentangled AGN and star formation radiative processes in the infrared spectrum for 703 IR bright X-ray AGN using multi-component SED fitting code, SED3FIT (Berta et al. 2013), and determined the AGN-corrected integrated rest-frame infrared luminosity attributed to star formation, total infrared AGN luminosity, AGN dust covering factors and AGN fractions. Our main results can be summarized as follows:
- We find flat trends consistent with other literature when averaging $\mathrm{L}_{\mathrm{IR}}^{\mathrm{SF}}$ in bins of bolometric AGN luminosity for less powerful AGN ( $\mathrm{L}_{\mathrm{AGN}}<$ $\left.10^{45} \mathrm{erg} \mathrm{s}^{-1}\right)$, as well as the stronger correlations found when averaging $\mathrm{L}_{\mathrm{X}}$ in bins of star formation activity for AGN at low redshifts $(0.1<z<0.8)$.

- We further decompose AGN SEDs to isolate the dusty torus component in the IR and compare to the bolometric AGN luminosity to estimate nuclear obscuration. We determine an average dust covering factor slightly lower than other literature at $\mathrm{CF}=33 \%$, which indicates a Type 2 (obscured) population of roughly a third. Further investigation of X-ray hardness reveals several X-ray AGN with covering factors that contradict the expected nuclear obscuration determined by hardness ratios (e.g. high covering factor with a low hardness ratio that is indicative of an unobscured central engine), providing further evidence that observational differences between AGN types are not primarily driven by line-of-sight dusty torus inclination.

- We uncover a wide range in the fraction of infrared luminosity attributed to AGN activity across all redshifts, and determine no statistically significant trend exists when evaluating $f_{\text {AGN }}$ as a function of total infrared, X-ray or $24 \mu \mathrm{m}$ luminosity. The mean $f_{\text {AGN }}$ as a function of rest-frame IR wavelength shows peak AGN contamination lives in the mid-IR range and becomes insignificant at wavelengths larger than $\sim 30 \mu \mathrm{m}$, but the sample dispersion is large $( \pm 20-30 \%)$ at all wavelengths. These results demonstrate the importance of SED decomposition for individual AGN and host galaxies in order to accurately quantify AGN contamination in the IR, particularly prior to using IR photometry to estimate host galaxy properties.

While considering all of the implications discussed in this paper, we should remember that current FIR detections of intermediate and high redshift X-ray AGN in star-forming galaxies are limited by the sensitivity of far-infrared and submillimeter observatories like the Herschel Space Observatory. The currently available resolutions limit us to the most powerful star-forming systems and we need deeper, more sensitive observations to capture the dust properties of AGN that reside in smaller and/or quiescent galaxies in order to complete the evolutionary picture.

AB would like to thank Susan Terebey for support and advisement. This research was supported in part by NASA grants NNX15AQ06A and NNX16AF38G, research from Hubble Space Telescope programs HST-GO14083.002-A and HST-GO-13718.002-A, and NSF grant AST-131331. This research has made use of data from HerMES project. HerMES is a Herschel Key Programme utilizing Guaranteed Time from the SPIRE instrument team, ESAC scientists and a mission scientist. The HerMES data was accessed through the Herschel Database in Marseille (HeDaM - http://hedam.lam.fr) operated by CeSAM and hosted by the Laboratoire d'Astrophysique de Marseille. HerMES DR3 was made possible through 
support of the Herschel Extragalactic Legacy Project, HELP (http://herschel.sussex.ac.uk).

\section{REFERENCES}

Aird, J., Nandra, K., Laird, E. S., et al. 2010, MNRAS, 401, 2531

Aird, J., Coil, A. L., Moustakas, J., et al. 2012, ApJ, 746, 90

Alexander, D. M., \& Hickox, R. C. 2012, ??jnlNewAR, 56, 93

Alexander, D. M., Bauer, F. E., Brandt, W. N., et al. 2003, AJ, 125,383

Antonucci, R. 1993, ARA\&A, 31, 473

Ashby, M. L. N., Stern, D., Brodwin, M., et al. 2009, ApJ, 701, 428

Azadi, M., Aird, J., Coil, A. L., et al. 2015, ApJ, 806, 187

Azadi, M., Coil, A. L., Aird, J., et al. 2017, ApJ, 835, 27

Barmby, P., Alonso-Herrero, A., Donley, J. L., et al. 2006, ApJ, 642, 126

Beckmann, V., Soldi, S., Ricci, C., et al. 2009, A\&A, 505, 417

Berta, S., Lutz, D., Santini, P., et al. 2013, AAP, 551, A100

Bianchi, S., Maiolino, R., \& Risaliti, G. 2012, Advances in Astronomy, 2012, 782030

Blandford, R. D., \& Payne, D. G. 1982, MNRAS, 199, 883

Bonfield, D. G., Jarvis, M. J., Hardcastle, M. J., et al. 2011, MNRAS, 416, 13

Brand, K., Brown, M. J., Dey, A., et al. 2006, ApJ, 641, 140

Brandt, W. N., \& Alexander, D. M. 2015, A\&A Rev., 23, 1

Brusa, M., Civano, F., Comastri, A., et al. 2010, ApJ, 716, 348

Casey, C. M., Narayanan, D., \& Cooray, A. 2014, Physics Reports, 541, 45

Chen, C.-T. J., Hickox, R. C., Alberts, S., et al. 2013, ApJ, 773, 3

-. 2015, ApJ, 802, 50

Ciesla, L., Charmandaris, V., Georgakakis, A., et al. 2015, A\&A, 576, A10

Civano, F., Marchesi, S., Comastri, A., et al. 2016, ApJ, 819, 62

Condon, J. J., Anderson, E., \& Broderick, J. J. 1995, AJ, 109, 2318

Cowley, M. J., Spitler, L. R., Tran, K.-V. H., et al. 2016 , MNRAS, 457, 629

Croton, D. J., Springel, V., White, S. D. M., et al. 2006, MNRAS 365, 11

da Cunha, E., Charlot, S., \& Elbaz, D. 2008, MNRAS, 388, 1595

da Cunha, E., Walter, F., Smail, I. R., et al. 2015, ApJ, 806, 110

Daddi, E., Alexander, D. M., Dickinson, M., et al. 2007, ApJ, 670, 173

Dai, Y. S., Wilkes, B. J., Bergeron, J., et al. 2017, ArXiv e-prints, arXiv: 1511.06761

Davies, R. I., Burtscher, L., Rosario, D., et al. 2015, ApJ, 806, 127

Dekel, A., Birnboim, Y., Engel, G., et al. 2009, Nature, 457, 451

Del Moro, A., Alexander, D. M., Mullaney, J. R., et al. 2013, A\&A, 549, A59

Delvecchio, I., Lutz, D., Berta, S., et al. 2015, MNRAS, 449, 373

Di Matteo, T., Springel, V., \& Hernquist, L. 2005, Nature, 433, 604

Diamond-Stanic, A. M., Moustakas, J., Tremonti, C. A., et al. 2012, ApJ, 755, L26

Dijkstra, M., \& Loeb, A. 2009, MNRAS, 400, 1109

Donley, J. L., Rieke, G. H., Rigby, J. R., \& Pérez-González, P. G. 2005, ApJ, 634, 169

Donley, J. L., Koekemoer, A. M., Brusa, M., et al. 2012, ApJ, 748, 142

Ellison, S. L., Mendel, J. T., Patton, D. R., \& Scudder, J. M. 2013, MNRAS, 435, 3627

Ellison, S. L., Teimoorinia, H., Rosario, D. J., \& Mendel, J. T. 2016, MNRAS, 458, L34

Ezhikode, S. H., Gandhi, P., Done, C., et al. 2016, ArXiv e-prints, arXiv: 1610.00429 [astro-ph.HE]

Fabian, A. C. 2012, ARA\&A, 50, 455

Fazio, G. G., Hora, J. L., Allen, L. E., et al. 2004, ApJ Supplement Series, 154, 10

Ferrarese, L., \& Merritt, D. 2000, ApJ, 539, L9

Fiore, F., Grazian, A., Santini, P., et al. 2008, ApJ, 672, 94

Fiore, F., Puccetti, S., Brusa, M., et al. 2009, ApJ, 693, 447

Fritz, J., Franceschini, A., \& Hatziminaoglou, E. 2006, MNRAS, 366,767

Gabor, J. M., \& Bournaud, F. 2014, MNRAS, 441, 1615
Geach, J. E., Hickox, R. C., Diamond-Stanic, A. M., et al. 2014, Nature, 516, 68

Gonzalez, A. H., Brodwin, M., Brown, M. J. I., et al. 2010, in American Astronomical Society Meeting Abstracts, Vol. 216 American Astronomical Society Meeting Abstracts \#216, 415.13

Goulding, A. D., Alexander, D. M., Bauer, F. E., et al. 2012, ApJ, 755,5

Green, P. J., Silverman, J. D., Cameron, R. A., et al. 2004, The Astrophysical Journal Supplement Series, 150, 43

Griffin, M. J., Abergel, A., Abreu, A., et al. 2010, A\&A, 518, L3

Gültekin, K., Richstone, D. O., Gebhardt, K., et al. 2009, ApJ, 698,198

Haardt, F., \& Maraschi, L. 1991, ApJ, 380, L51

Haas, M., Klaas, U., Müller, S. A. H., et al. 2003, A\&A, 402, 87

Harrison, C. M., Alexander, D. M., Mullaney, J. R., \& Swinbank, A. M. 2014, MNRAS, 441, 3306

Hickox, R. C., \& Alexander, D. M. 2018, ArXiv e-prints, arXiv: 1806.04680

Hickox, R. C., Mullaney, J. R., Alexander, D. M., et al. 2014, ApJ, 782, 9

Hickox, R. C., Jones, C., Forman, W. R., et al. 2007, ApJ, 671, 1365

-. 2009, ApJ, 696, 891

Ho, L. C. 2008, ARA\&A, 46, 475

Hopkins, P. F., Hayward, C. C., Narayanan, D., \& Hernquist, L. 2012, MNRAS, 420, 320

Hopkins, P. F., \& Hernquist, L. 2009, ApJ, 694, 599

Hopkins, P. F., Hernquist, L., Cox, T. J., et al. 2006, ApJS, 163, 1

Jannuzi, B. T., \& Dey, A. 1999, in Astronomical Society of the Pacific Conference Series, Vol. 191, Photometric Redshifts and the Detection of High Redshift Galaxies, ed. R. Weymann, L. Storrie-Lombardi, M. Sawicki, \& R. Brunner, 111

Jorstad, S. G., Marscher, A. P., Lister, M. L., et al. 2005, AJ, 130,1418

Kauffmann, G., \& Haehnelt, M. 2000, MNRAS, 311, 576

Kenter, A., Murray, S. S., Forman, W. R., et al. 2005, ApJS, 161, 9

Kirkpatrick, A., Pope, A., Alexander, D. M., et al. 2012, ApJ, 759, 139

Kirkpatrick, A., Alberts, S., Pope, A., et al. 2017, ApJ, 849, 111

Kochanek, C. S., Eisenstein, D. J., Cool, R. J., et al. 2012, ApJS, 200,8

Kormendy, J., \& Ho, L. C. 2013, Annual Review of A\&A, 51, 511

Kormendy, J., \& Richstone, D. 1995, ARA\&A, 33, 581

Lanzuisi, G., Piconcelli, E., Fiore, F., et al. 2009, A\&A, 498, 67

Lanzuisi, G., Ranalli, P., Georgantopoulos, I., et al. 2015, A\&A. 573, A 137

Lanzuisi, G., Delvecchio, I., Berta, S., et al. 2017, ArXiv e-prints, arXiv: 1702.07357

Lapi, A., Raimundo, S., Aversa, R., et al. 2014, ApJ, 782, 69

Lawrence, A. 1991, MNRAS, 252, 586

Lawrence, A., \& Elvis, M. 2010, ArXiv e-prints, arXiv:1002.1759 [astro-ph.GA]

Lehmer, B. D., Alexander, D. M., Bauer, F. E., et al. 2010, ApJ 724,559

Leung, G. C. K., Coil, A. L., Azadi, M., et al. 2017, ApJ, 849, 48

Lusso, E., Comastri, A., Simmons, B. D., et al. 2012, MNRAS, 425,623

Lutz, D., Sturm, E., Tacconi, L. J., et al. 2008, ApJ, 684, 853

Magorrian, J., Tremaine, S., Richstone, D., et al. 1998, AJ, 115, 2285

Maiolino, R., Shemmer, O., Imanishi, M., et al. 2007, A\&A, 468, 979

Marconi, A., \& Hunt, L. K. 2003, ApJ, 589, L21

Mateos, S., Carrera, F. J., Alonso-Herrero, A., et al. 2015, MNRAS, 449, 1422

-. 2016, ApJ, 819, 166

Mateos, S., Carrera, F. J., Barcons, X., et al. 2017, ApJ, 841, L18

Mendez, A. J., Coil, A. L., Aird, J., et al. 2013, ApJ, 770, 40

Merloni, A., Bongiorno, A., Brusa, M., et al. 2014, MNRAS, 437, 3550

Miley, G. 1980, ARA\&A, 18, 165

Mineo, S., Gilfanov, M., \& Sunyaev, R. 2012a, MNRAS, 419, 2095 —. 2012b, MNRAS, 426, 1870

Mullaney, J. R., Alexander, D. M., Goulding, A. D., \& Hickox,

R. C. 2011, MNRAS, 414, 1082 
Mullaney, J. R., Pannella, M., Daddi, E., et al. 2012, MNRAS, 419, 95

Mullaney, J. R., Alexander, D. M., Aird, J., et al. 2015, MNRAS, 453, L83

Murray, S. S., Kenter, A., Forman, W. R., et al. 2005, ApJS, 161, 1

Mushotzky, R. 2004, in Astrophysics and Space Science Library, Vol. 308, Supermassive Black Holes in the Distant Universe, ed. A. J. Barger, 53

Nandra, K., \& Pounds, K. A. 1994, MNRAS, 268, 405

Nardini, E., Risaliti, G., Salvati, M., et al. 2008, MNRAS, 385, L130

Nenkova, M., Sirocky, M. M., Ivezić, Ž., \& Elitzur, M. 2008a, ApJ, 685, 147

Nenkova, M., Sirocky, M. M., Nikutta, R., Ivezić, Ž., \& Elitzur, M. 2008b, ApJ, 685, 160

Netzer, H. 2009, MNRAS, 399, 1907

-. 2015, ARA\&A, 53, 365

Netzer, H., \& Trakhtenbrot, B. 2007, ApJ, 654, 754

Oliver, S. J., Bock, J., Altieri, B., et al. 2012, MNRAS, 424, 1614

Omont, A., Cox, P., Bertoldi, F., et al. 2001, A\&A, 374, 371

Pilbratt, G. L., Riedinger, J. R., Passvogel, T., et al. 2010, A\&A, 518, L1

Poglitsch, A., Waelkens, C., Geis, N., et al. 2010, A\&A, 518, L2

Ranalli, P., Comastri, A., \& Setti, G. 2003, A\&A, 399, 39

Reeves, J. N., O'Brien, P. T., Braito, V., et al. 2009, ApJ, 701, 493

Ricci, C., Trakhtenbrot, B., Koss, M. J., et al. 2017, Nature, 549, 488

Rieke, G. H., Young, E. T., Engelbracht, C. W., et al. 2004, ApJ Supplement Series, 154, 25

Rosario, D. J., Santini, P., Lutz, D., et al. 2012, AAP, 545, A45

Rosario, D. J., McIntosh, D. H., van der Wel, A., et al. 2015, A\&A, 573, A85

Roseboom, I. G., Oliver, S. J., Kunz, M., et al. 2010, MNRAS, 409, 48

Rowan-Robinson, M., Valtchanov, I., \& Nandra, K. 2009, MNRAS, 397, 1326

Rupke, D. S. N., \& Veilleux, S. 2011, ApJ, 729, L27

Scoville, N., Aussel, H., Brusa, M., et al. 2007, ApJS, 172, 1

Shankar, F. 2009, ??jnlNewAR, 53, 57

Shields, G. A., \& Salviander, S. 2009, in Astronomical Society of the Pacific Conference Series, Vol. 419, Galaxy Evolution: Emerging Insights and Future Challenges, ed. S. Jogee,

I. Marinova, L. Hao, \& G. A. Blanc, 392

Shimizu, T. T., Mushotzky, R. F., Meléndez, M., et al. 2017, MNRAS, 466, 3161
Siebenmorgen, R., Heymann, F., \& Efstathiou, A. 2015, A\&A, 583, A120

Silk, J., \& Nusser, A. 2010, ApJ, 725, 556

Smith, D. J. B., Jarvis, M. J., Lacy, M., \& Martínez-Sansigre, A. 2008, MNRAS, 389, 799

Somerville, R. S., Hopkins, P. F., Cox, T. J., Robertson, B. E., \& Hernquist, L. 2008, MNRAS, 391, 481

Stalevski, M., Ricci, C., Ueda, Y., et al. 2016, MNRAS, 458, 2288

Stanley, F., Harrison, C. M., Alexander, D. M., et al. 2015,

MNRAS, 453, 591

Stern, D. 2015, ApJ, 807, 129

Stern, D., Eisenhardt, P., Gorjian, V., et al. 2005, ApJ, 631, 163

Stevens, J. A., Jarvis, M. J., Coppin, K. E. K., et al. 2010 , MNRAS, 405, 2623

Sturm, E., González-Alfonso, E., Veilleux, S., et al. 2011, ApJ, 733, L16

Symeonidis, M., Giblin, B. M., Page, M. J., et al. 2016, MNRAS, 459,257

Treister, E., Krolik, J. H., \& Dullemond, C. 2008, ApJ, 679, 140

Treister, E., Schawinski, K., Urry, C. M., \& Simmons, B. D. 2012 , ApJ, 758, L39

Treister, E., Urry, C. M., Chatzichristou, E., et al. 2004, ApJ, 616,123

Treister, E., Cardamone, C. N., Schawinski, K., et al. 2009, ApJ, 706,535

Tristram, K. R. W., Meisenheimer, K., Jaffe, W., et al. 2007, A\&A, 474, 837

Tristram, K. R. W., Raban, D., Meisenheimer, K., et al. 2009, A\&A. 502, 67

Urry, C. M., \& Padovani, P. 1995, PASP, 107, 803

Vignali, C., Brandt, W. N., Schneider, D. P., \& Kaspi, S. 2005, AJ, 129, 2519

Volonteri, M. 2010, The A\&A Review, 18, 279

Volonteri, M., Capelo, P. R., Netzer, H., et al. 2015, MNRAS, 452, L6

Volonteri, M., Haardt, F., \& Madau, P. 2003, ApJ, 582, 559

Weisskopf, M. C., Brinkman, B., Canizares, C., et al. 2002, PASP, 114, 1

Werner, M. W., Roellig, T. L., Low, F. J., et al. 2004, ApJS, 154, 1

Winter, L. M., Mushotzky, R. F., Reynolds, C. S., \& Tueller, J. 2009, ApJ, 690, 1322

Xue, Y. Q., Brandt, W. N., Luo, B., et al. 2010, ApJ, 720, 368 


\section{APPENDIX - SAMPLE EXTREME SEDS}

Below are sample SEDs of AGN that reside in the more extreme regions of Figure 9, marked by black crosses. Figure 13 shows two objects with X-ray HRs indicative of an unobscured nucleus with little to no dust or gas absorbing their $\mathrm{X}$-ray luminosities. However, the object on the left has a high dusty torus covering factor, which is contradictory to what we'd expect to see based on the HR and the inclination-based AGN unification model (Antonucci 1993; Urry \& Padovani 1995). Similarly, in Figure 14 we see two objects with HRs that signify the presence of highly obscuring column densities, but SED decomposition for the object on the right determined a low covering factor that contradicts the HR estimate. These objects support the need for a different perspective on what truly drives the observational differences between AGN classifications.
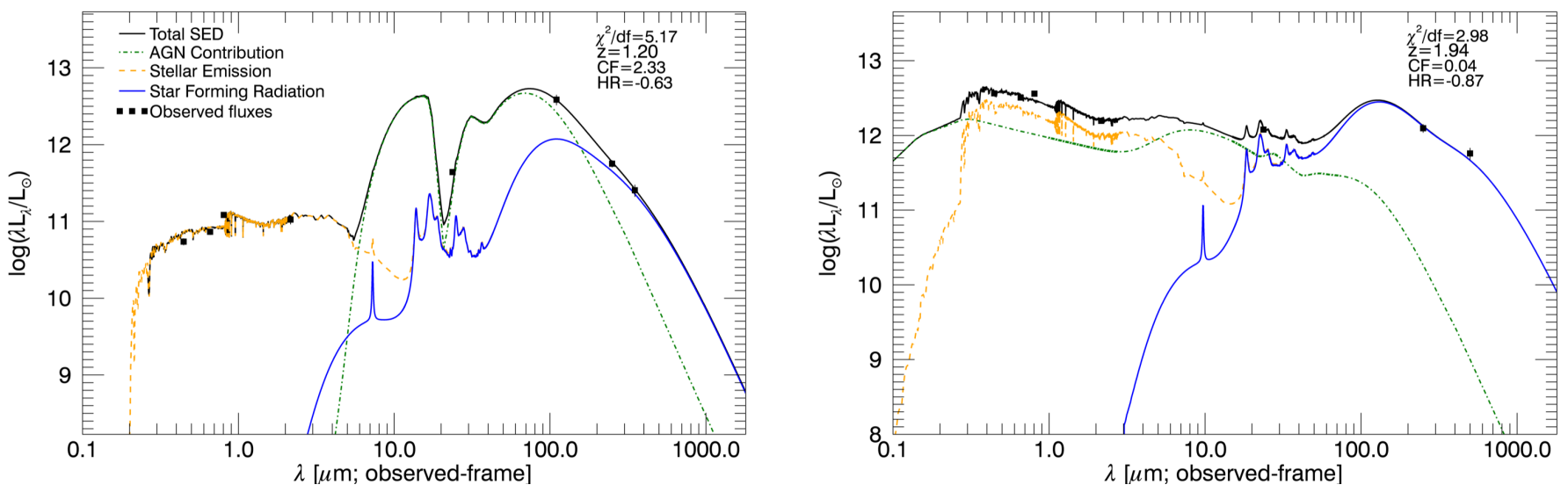

Figure 13. Generated example 'extreme' spectral energy distributions of AGN with hardness ratios $\lesssim-0.5$, indicative of an unobscured nucleus with with little to no obscuring dust and gas. Left: SED of an X-ray unobscured AGN with high covering factor. Right: SED of an X-ray unobscured AGN with a low covering factor.
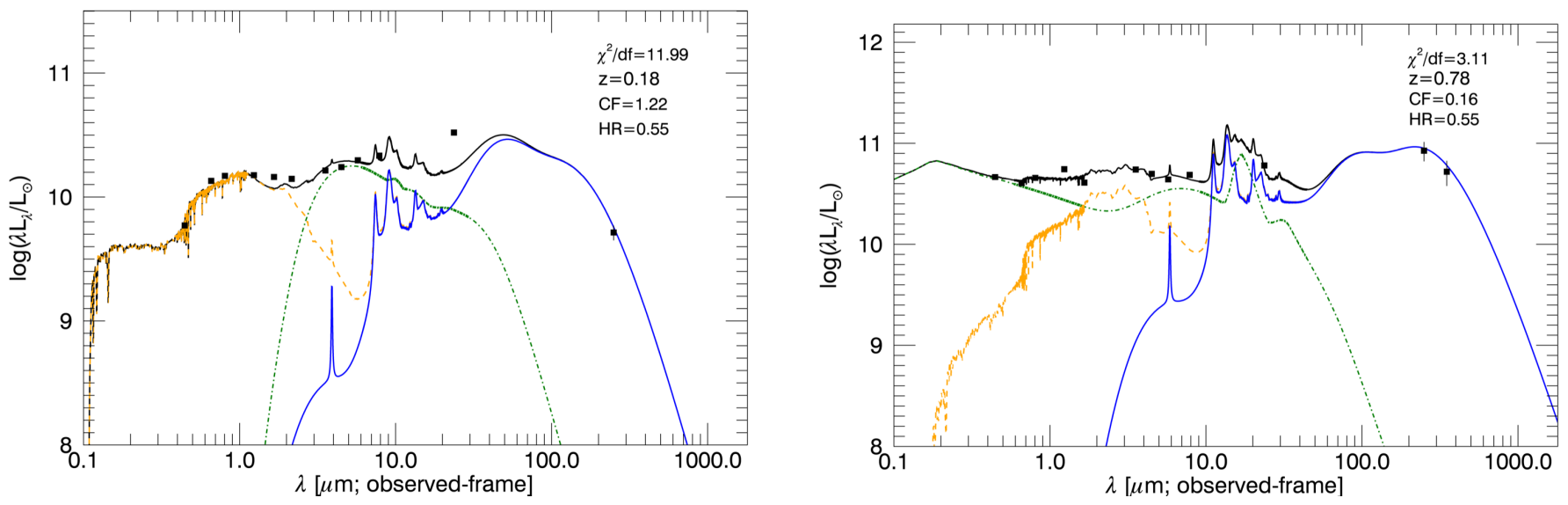

Figure 14. Generated example 'extreme' spectral energy distributions of AGN with hardness ratios $\gtrsim 0.5$, indicative of an obscured nucleus with thick absorbing circumnuclear gas and dust. Left: SED of an X-ray obscured AGN with high covering factor. Right: SED of an X-ray obscured AGN with a low covering factor. 